\title{
NATIONAL ACADEMY OF SCIENCES
}

\author{
July 1, 1964 \\ OFFICERS
}

\author{
President-Frederick Seitz \\ Vice President-J. A. Stratton \\ Home Secretary - Hugh L. Dryden \\ Foreign Secretary-Harrison Brown \\ Treasurer-L. V. Berkner \\ Executive Officer \\ S. D. Cornell
}

\author{
Term expires \\ June 30, 1966 \\ June 30, 1965 \\ June 30, 1967 \\ June 30, 1966 \\ June 30, 1968 \\ Business Manager \\ G. D. Meid
}

\section{COUNCIL}

*Berkner, L. V.

*Brown, Harrison

*Dryden, Hugh L.

Kornberg, Arthur

*Pitzer, K. S.

*Revelle, Roger
(1968)

(1965)

\author{
*Seitz, Frederick \\ Sonneborn, T. M. \\ *Stratton, J. A. \\ Weiss, Paul \\ Wood, W. Barry, Jr.
}

(1966)

\section{MEMBERS}

The number in parentheses, following year of election, indicates the Section to which the member belongs, as follows:
(1) Mathematics
(2) Astronomy
(3) Physics
(4) Engineering
(5) Chemistry
(6) Geology
(7) Botany

(8) Zoology and Anatomy

(9) Physiology

(10) Pathology and Microbiology

(11) Anthropology

(12) Psychology

(13) Geophysics

(14) Biochemistry

Abbot, Charles Greeley, 1915 (2), Smithsonian Institution, Washington, D. C. 20560

Abelson, Philip Hauge, 1959 (6), Geophysical Laboratory, Carnegie Institution of Washington, 2801 Upton Street, N. W., Washington, D. C. 20008

Adams, Leason Heberling, 1943 (13), Institute of Geophysics, University of California, Los Angeles, California 90024

Adams, Roger, 1929 (5), Department of Chemistry and Chemical Engineering, University of Illinois, Urbana, Illinois 61803

Ahlfors, Lars Valerian, 1953 (1), Department of Mathematics, Harvard University, 2 Divinity Avenue, Cambridge, Massachusetts 02138

Albert, Abraham Adrian, 1943 (1), 111 Eckhart Hall, University of Chicago, 1118 East 58th Street, Chicago, Illinois 60637

Albright, William Foxwell, 1955 (11), Oriental Seminary, Johns Hopkins University, Baltimore, Maryland 21218

\footnotetext{
* Members of the Executive Committee of the Council of the Academy.
} 
Alexander, James Waddell, 1930 (1), 29 Cleveland Lane, Princeton, New Jersey 08541

Allen, Eugene Thomas, 1930 (6), 135 Pleasant Street, Arlington, Massachusetts 02174

Aller, Lawrence Hugh, 1962 (2), Department of Astronomy, University of California, Los Angeles, California 90024

Allison, Samuel King, 1946 (3), The Enrico Fermi Institute for Nuclear Studies, University of Chicago, Chicago, Illinois 60637

Alvarez, Luis Walter, 1947 (3), Lawrence Radiation Laboratory, University of California, Berkeley, California 94720

Anderson, Carl David, 1938 (3), California Institute of Technology, Pasadena, California 91109

Anderson, Charles Alfred, 1957 (6), United States Geological Survey, Department of the Interior, Washington, D. C. 20240

Anderson, Edgar, 1954 (7), Missouri Botanical Garden, 2315 Tower Grove Avenue, St. Louis, Missouri 63110

Anderson, Herbert Lawrence, 1960 (3), The Enrico Fermi Institute for Nuclear Studies, University of Chicago, Chicago, Illinois 60637

Anderson, Thomas Foxen, 1964 (7), The Institute for Cancer Research, 7701 Burholme Avenue, Philadelphia, Pennsylvania 19111

Anfinsen, Christian Boehmer, 1963 (14), Laboratory of Chemical Biology, National Institute of Arthritis and Metabolic Diseases, National Institutes of Health, Bethesda, Maryland 20014

Armstrong, Charles, 1944 (10), National Institutes of Health, Bethesda, Maryland 20014

Arnold, James Richard, 1964 (13), Department of Chemistry, University of California, San Diego, La Jolla, California 92038

Arnold, William Archibald, 1962 (7), Biology Division, Oak Ridge National Laboratory, P. O. Box Y, Oak Ridge, Tennessee 37831

Arnon, Daniel Israel, 1961 (7), Department of Cell Physiology, 251 Hilgard Hall, University of California, Berkeley, California 94720

Astin, Allen Varley, 1960 (4), National Bureau of Standards, Washington, D. C. 20234

Astwood, Edwin Bennett, 1957 (9), New England Center Hospital, Harrison Avenue and Bennet Street, Boston, Massachusetts 02111

Aub, Joseph Charles, 1957 (9), Massachusetts General Hospital, Fruit Street, Boston, Massachusetts 02114

Babcock, Harold Delos, 1933 (2), 1820 Atchison Street, Pasadena, California 91104

Babcock, Horace Welcome, 1954 (2), Mount Wilson and Palomar Observatories, 813 Santa Barbara Street, Pasadena, California 91106

Bacher, Robert Fox, 1947 (3), California Institute of Technology, Pasadena, California 91109

Badger, Richard McLean, 1952 (5), Gates and Crellin Laboratories of Chemistry, California Institute of Technology, Pasadena, California 91109

Bailey, Percival, 1953 (10), Illinois State Psychiatric Institute, 1601 West Taylor Street, Chicago, Illinois 60612

Bain, Edgar Collins, 1954 (4), 434 Maple Lane, Edgeworth, Sewickley, Pennsylvania 15143

Bainbridge, Kenneth Tompkins, 1946 (3), Department of Physics, Harvard University, Cambridge, Massachusetts 02138 
Baker, William Oliver, 1961 (5), Bell Telephone Laboratories, Incorporated, Murray Hill, New Jersey 07971

Ball, Eric Glendinning, 1948 (14), Department of Biological Chemistry, Harvard Medical School, 25 Shattuck Street, Boston, Massachusetts 02115

Balls, Arnold Kent, 1954 (14), 1988 Thousand Oaks Boulevard, Berkeley, California 94707

Bard, Philip, 1944 (9), School of Medicine, Johns Hopkins University, 725 North Wolfe Street, Baltimore, Maryland 21205

Bardeen, John, 1954 (3), Department of Physics, University of Illinois, Urbana, Illinois 61803

Barker, Horace Albert, 1953 (7), Department of Biochemistry, Biochemistry Building, University of California, Berkeley, California 94720

Bartelmez, George William, 1949 (8), 224 Agnes Avenue, Missoula, Montana 59801

Bartlett, Paul Doughty, 1947 (5), Department of Chemistry, Harvard University, 12 Oxford Street, Cambridge, Massachusetts 02138

Beach, Frank Ambrose, 1949 (12), Department of Psychology, University of California, Berkeley, California 94720

Beadle, George Wells, 1944 (7), University of Chicago, 5801 Ellis Avenue, Chicago, Illinois 60637

Beams, Jesse Wakefield, 1943 (3), Physics Laboratory, University of Virginia, McCormick Road, Charlottesville, Virginia 22903

Benedict, Manson, 1956 (4), Department of Nuclear Engineering, Massachusetts Institute of Technology, Cambridge, Massachusetts 02139

Benioff, Victor Hugo, 1953 (13), Seismological Laboratory, California Institute of Technology, 220 North San Rafael Avenue, Pasadena, California 91105

Benzer, Seymour, 1961 (7), Department of Biological Sciences, Purdue University, Lafayette, Indiana 47907

Berkner, Lloyd Viel, 1948 (13), Graduate Research Center of the Southwest, P. O. Box 8478, Dallas, Texas 75205

Bers, Lipman, 1964 (1), Department of Mathematics, Columbia University, New York, New York 10027

Bethe, Hans Albrecht, 1944 (3), Laboratory of Nuclear Studies, Cornell University, Ithaca, New York 14850

Bigelow, Henry Bryant, 1931 (8), Museum of Comparative Zoology at Harvard College, Oxford Street, Cambridge, Massachusetts 02138

Birch, Albert Francis, 1950 (6), Department of Geological Sciences, Harvard University, Cambridge, Massachusetts 02138

Birge, Raymond Thayer, 1932 (3), Department of Physics, University of California, Berkeley, California 94720

Bjerknes, Jacob, 1947 (13), Department of Meteorology, University of California, Los Angeles, California 90024

Blackwelder, Eliot, 1936 (6), P. O. Box 6506, Stanford, California 94305

Blalock, Alfred, 1945 (10), School of Medicine, Johns Hopkins University, 725 North Wolfe Street, Baltimore, Maryland 21205

Bleakney, Walker, 1959 (3), Palmer Physical Laboratory, Princeton University, P. O. Box 708, Princeton, New Jersey 08540

Blinks, Lawrence Rogers, 1955 (7), Hopkins Marine Station of Stanford University, Pacific Grove, California 93950

Bloch, Felix, 1948 (3), Department of Physics, Stanford University, Stanford, California 94305 
Bloch, Konrad Emil, 1956 (14), Department of Chemistry, Harvard University, 12 Oxford Street, Cambridge, Massachusetts 02138

Bloembergen, Nicolaas, 1960 (3), Pierce Hall, Harvard University, Cambridge, Massachusetts 02138

Blomquist, Alfred Theodore, 1960 (5), Department of Chemistry, Cornell University, Ithaca, New York 14850

Bloom, William, 1954 (8), University of Chicago, 5640 South Ellis Avenue, Chicago, Illinois 60637

Bochner, Salomon, 1950 (1), Department of Mathematics, Princeton University, P. O. Box 708, Princeton, New Jersey 08540

Bode, Hendrik Wade, 1957 (4), Bell Telephone Laboratories, Incorporated, Whippany, New Jersey 07981

Bodenstein, Dietrich H. F. A., 1958 (8), Department of Biology, University of Virginia, Charlottesville, Virginia 22903

Bodian, David, 1958 (8), Department of Anatomy, School of Medicine, Johns Hopkins University, 725 North Wolfe Street, Baltimore, Maryland 21205

Boekelheide, Virgil Carl, 1962 (5), Department of Chemistry, University of Oregon, Eugene, Oregon 97403

Bolton, Elmer K., 1946 (5), 2310 West 11th Street, Wilmington, Delaware 19805

Bonner, James Frederick, 1950 (7), Division of Biology, California Institute of Technology, Pasadena, California 91109

Booker, Henry George, 1960 (13), School of Electrical Engineering, Cornell University, Ithaca, New York 14850

Boring, Edwin Garrigues, 1932 (12), Memorial Hall, Harvard University, Cambridge, Massachusetts 02138

Borthwick, Harry Alfred, 1961 (7), Plant Industry Station, United States Department of Agriculture, Beltsville, Maryland 20251

Bott, Raoul, 1964 (1), Department of Mathematics, Harvard University, 2 Divinity Avenue, Cambridge, Massachusetts 02138

Bowen, Ira Sprague, 1936 (2), Mount Wilson and Palomar Observatories, 813 Santa Barbara Street, Pasadena, California 91106

Bradbury, Norris Edwin, 1951 (3), Los Alamos Scientific Laboratory, P. O. Box 1663, Los Alamos, New Mexico 87544

Bradley, Wilmot Hyde, 1946 (6), United States Geological Survey, Department of the Interior, Washington, D. C. 20240

Braidwood, Robert John, 1964 (11), The Oriental Institute, University of Chicago, Chicago, Illinois 60637

Bramlette, Milton Nunn, 1954 (6), Scripps Institution of Oceanography, La Jolla, California 92038

Brattain, Walter Houser, 1959 (3), Bell Telephone Laboratories, Incorporated, Murray Hill, New Jersey 07971

Brauer, Richard Dagobert, 1955 (1), Department of Mathematics, Harvard University, 2 Divinity Avenue, Cambridge, Massachusetts 02138

Braun, Armin Charles, 1960 (7), Rockefeller Institute, New York, New York 10021

Breit, Gregory, 1939 (3), Sloane Physics Laboratory, Yale University, P. O. Box 2014, New Haven, Connecticut 06520

Brewer, Leo, 1959 (5), Department of Chemistry, University of California, Berkeley, California 94720

Briggs, Robert William, 1962 (8), Department of Zoology, Indiana University, Bloomington, Indiana 47405 
Brillouin, Leon, 1953 (3), 88 Central Park West, New York, New York 10023

Brink, Frank, Jr., 1959 (9), Rockefeller Institute, New York, New York 10021

Brink, Royal Alexander, 1947 (7), Department of Genetics, University of Wisconsin, Madison, Wisconsin 53706

Brode, Robert Bigham, 1949 (3), Department of Physics, University of California, Berkeley, California 94720

Brode, Wallace Reed, 1954 (5), 3900 Connecticut Avenue, Washington, D. C. 20008

Bronk, Detlev Wulf, 1939 (9), Rockefeller Institute, New York, New York 10021

Brooks, Harvey, 1962 (4), 217 Pierce Hall, Harvard University, Cambridge, Massachusetts 02138

Brouwer, Dirk, 1951 (2), Yale University Observatory, Box 2023, Yale Station, New Haven, Connecticut 06520

Brown, Harrison Scott, 1955 (13), Division of Geological Sciences, California Institute of Technology, Pasadena, California 91109

Brown, Herbert Charles, 1957 (5), Department of Chemistry, Purdue University, Lafayette, Indiana 47907

Buchanan, John Machlin, 1962 (14), Division of Biochemistry, Department of Biology, Massachusetts Institute of Technology, Cambridge, Massachusetts 02139

Bucher, Walter Hermann, 1938 (6), Department of Geology, Columbia University, New York, New York 10027

Buddington, Arthur Francis, 1943 (6), Department of Geology, Princeton University, Princeton, New Jersey 08540

Buerger, Martin Julian, 1953 (6), Department of Geology and Geophysics, Massachusetts Institute of Technology, Cambridge, Massachusetts 02139

Bullock, Theodore Holmes, 1963 (8), Department of Zoology, University of California, Los Angeles, California 90024

Burkholder, Paul Rufus, 1949 (7), Lamont Geological Observatory of Columbia University, Torrey Cliff, Palisades, New York 10964

Burns, Robert Kyle, 1955 (8), Department of Biology, Bridgewater College, Bridgewater, Virginia 22812

Burris, Robert Harza, 1961 (7), Department of Biochemistry, University of Wisconsin, Madison, Wisconsin 53706

Bush, Vannevar, 1934 (4), Massachusetts Institute of Technology, Cambridge, Massachusetts 02139

Byerly, Perry, 1946 (13), Seismographic Stations, University of California, Berkeley, California 94720

Byers, Horace Robert, 1952 (13), Department of Geophysical Sciences, University of Chicago, Chicago, Illinois 60637

Calvin, Melvin, 1954 (5), Laboratory of Chemical Biodynamics, University of California, Berkeley, California 94720

Cannon, Paul Roberts, 1946 (10), Box 56, Route 2, Yorkville, Illinois 60560

Carmichael, Leonard, 1943 (12), National Geographic Society, 17th and M Streets, N. W., Washington, D. C. 20036

Carter, Herbert Edmund, 1953 (4), Department of Chemistry and Chemical Engineering, University of Illinois, Urbana, Illinois 61803

Castle, William Bosworth, 1939 (10), Boston City Hospital, Boston, Massachusetts 02118

Chamberlain, Owen, 1960 (3), Department of Physics, University of California, Berkeley, California 94720 
Chance, Britton, 1954 (14), Johnson Research Foundation, University of Pennsylvania, Philadelphia, Pennsylvania 19104

Chandler, William Henry, 1943 (7), 341 South Almont Drive, Beverly Hills, California 90211

Chandrasekhar, Subrahmanyan, 1955 (2), Laboratory for Astrophysics and Space Research, 933 East 56th Street, Chicago, Illinois 60637

Chaney, Ralph Works, 1947 (6), Department of Paleontology, University of California, Berkeley, California 94720

Charney, Jule Gregory, 1964 (13), Department of Meteorology, Massachusetts Institute of Technology, Cambridge, Massachusetts 02139

Chern, Shiing-shen, 1961 (1), Department of Mathematics, University of California, Berkeley, California 94720

Chew, Geoffrey Foucar, 1962 (3), Department of Physics, University of California, Berkeley, California 94720

Chipman, John, 1955 (4), Room 16-402, Department of Metallurgy, Massachusetts Institute of Technology, Cambridge, Massachusetts 02139

Clarke, Hans Thacher, 1942 (14), 19 Ware Street, Cambridge, Massachusetts 02138

Clausen, Jens Christian, 1959 (7), Department of Plant Biology, Carnegie Institution of Washington, Stanford, California 94305

Cleland, Ralph Erskine, 1942 (7), Department of Botany, Indiana University, Bloomington, Indiana 47405

Clemence, Gerald Maurice, 1952 (2), Yale University Observatory, 2034 Yale Station, New Haven, Connecticut 06520

Cleveland, Lemuel Roscoe, 1952 (8), Department of Zoology, University of Georgia, Athens, Georgia 30602

Cloos, Ernst, 1950 (6), Department of Geology, Johns Hopkins University, Baltimore, Maryland 21218

Cloud, Preston Ercelle, Jr., 1961 (6), Department of Geology and Geophysics, University of Minnesota, Minneapolis, Minnesota 55455

Coggeshall, Lowell Thelwell, 1949 (10), University of Chicago, Chicago, Illinois 60637

Colbert, Edwin Harris, 1957 (8), American Museum of Natural History, Central Park West at 79th Street, New York, New York 10024

Cole, Kenneth Stewart, 1956 (9), Laboratory of Biophysics, National Institute of Neurological Diseases and Blindness, National Institutes of Health, Bethesda, Maryland 20014

Comroe, Julius Hiram, Jr., 1961 (9), Cardiovascular Research Institute, University of California Medical Center, San Francisco, California 94122

Conant, James Bryant, 1929 (5), Wachtelstrasse 7, Berlin-Dahlem, Germany

Condon, Edward Uhler, 1944 (3), 761 Cascade Avenue, Boulder, Colorado 80302

Connick, Robert Elwell, 1963 (5), College of Chemistry, 420 Latimer Hall, University of California, Berkeley, California 94720

Coon, Carleton Stevens, 1955 (11), 207 Concord Street, Gloucester, Massachusetts 01930

Coons, Albert Hewett, 1962 (10), Department of Bacteriology and Immunology, Harvard Medical School, 25 Shattuck Street, Boston, Massachusetts 02115

Cope, Arthur Clay, 1947 (5), Department of Chemistry, Massachusetts Institute of Technology, Cambridge, Massachusetts 02139

Cori, Carl Ferdinand, 1940 (14), School of Medicine, Washington University, Euclid Avenue and Kingshighway, St. Louis, Missouri 63110 
Corner, George Washington, 1940 (8), American Philosophical Society, 104 South Fifth Street, Philadelphia, Pennsylvania 19106

Couch, John Nathaniel, 1943 (7), University of North Carolina, Chapel Hill, North Carolina 27515

Courant, Richard, 1955 (1), New York University, 4 Washington Place, New York, New York 10003

Cournand, André Frederic, 1958 (9), Cardio-Pulmonary Laboratory (Columbia University Division), Bellevue Hospital, 462 First Avenue, New York, New York 10016

Craig, Lyman Creighton, 1950 (14), Rockefeller Institute, New York, New York 10021

Cram, Donald James, 1961 (5), Department of Chemistry, University of California, Los Angeles, California 90024

Crawford, Bryce, Jr., 1956 (5), Graduate School, University of Minnesota, Minneapolis, Minnesota 55455

Crow, James Franklin, 1961 (8), Department of Medical Genetics, University of Wisconsin, Madison, Wisconsin 53706

Curme, George Oliver, Jr., 1944 (4), Union Carbide Corporation, 270 Park Avenue, New York, New York 10017

Curtin, David Yarrow, 1964 (5), Department of Chemistry and Chemical Engineering, University of Illinois, Urbana, Illinois 61803

Dalldorf, Gilbert, 1955 (10), Sloan-Kettering Institute for Cancer Research, 145 Boston Post Road, Rye, New York 10580

Danforth, Charles Haskell, 1942 (8), Department of Anatomy, Stanford University, Stanford, California 94305

Daniels, Farrington, 1947 (5), Solar Energy Laboratory, University of Wisconsin, Madison, Wisconsin 53706

Darken, Lawrence Stamper, 1961 (4), United States Steel Corporation, Research Center, M.S.59, Monroeville, Pennsylvania 15146

Darlington, Philip Jackson, Jr., 1964 (8), Museum of Comparative Zoology at Harvard College, Oxford Street, Cambridge, Massachusetts 02138

Davidson, Norman Ralph, 1960 (5), Gates and Crellin Laboratories of Chemistry, California Institute of Technology, Pasadena, California 91109

Davis, Hallowell, 1948 (9), Central Institute for the Deaf, 818 South Euclid, St. Louis, Missouri 63110

Debye, Peter, (1931) 1947* (5), Baker Laboratory, Cornell University, Ithaca, New York 14850

Delbrück, Max, 1949 (7), California Institute of Technology, Pasadena, California 91109

Demerec, Milislav, 1946 (8), Department of Biology, Brookhaven National Laboratory, Upton, Long Island, New York 11973

Den Hartog, Jacob Pieter, 1953 (4), Department of Mechanical Engineering, Massachusetts Institute of Technology, Cambridge, Massachusetts 02139

Dennison, David Mathias, 1953 (3), Randall Laboratory of Physics, University of Michigan, Ann Arbor, Michigan 48104

Deutsch, Martin, 1958 (3), Laboratory for Nuclear Science, Massachusetts Institute of Technology, Cambridge, Massachusetts 02139

Dingle, John Holmes, 1958 (10), Department of Preventive Medicine, Wearn Research Building, University Hospitals, Cleveland, Ohio 44106

* Elected a foreign associate in 1931; became a naturalized citizen in 1946 and a member of the Academy in 1947. 
Djerassi, Carl, 1961 (5), Department of Chemistry, Stanford University, Stanford, California 94305

Dobzhansky, Theodosius, 1943 (8), Rockefeller Institute, New York, New York 10021

Doering, William von Eggers, 1961 (5), Yale University, 1901 A Yale Station, New Haven, Connecticut 06520

Doisy, Edward Adelbert, 1938 (14), St. Louis University School of Medicine, 1402 South Grand Boulevard, St. Louis, Missouri 63104

Doob, Joseph Leo, 1957 (1), Department of Mathematics, University of Illinois, Urbana, Illinois 61803

Doty, Paul Mead, 1957 (14), Department of Chemistry, Harvard University, 12 Oxford Street, Cambridge, Massachusetts 02138

Doudoroff, Michael, 1962 (14), Department of Bacteriology, University of California, Berkeley, California 94720

Douglas, Jesse, 1946 (1), City University of New York, The City College, Convent Avenue and 139th Street, New York, New York 10031

Dragstedt, Lester Reynold, 1950 (10), Department of Surgery, University of Florida, Gainesville, Florida 32603

Draper, Charles Stark, 1957 (4), Room 33-207, Department of Aeronautics and Astronautics, Massachusetts Institute of Technology, Cambridge, Massachusetts 02139

Dryden, Hugh Latimer, 1944 (4), National Aeronautics and Space Administration, 400 Maryland Avenue, S. W., Washington, D. C. 20546

Dubos, René Jules, 1941 (10), Rockefeller Institute, New York, New York 10021

DuBridge, Lee Alvin, 1943 (3), California Institute of Technology, Pasadena, California 91109

Dulbecco, Renato, 1961 (10), The Salk Institute for Biological Studies, Box 9499, San Diego, California 92109

DuMond, Jesse William Monroe, 1953 (3), Department of Physics, California Institute of Technology, Pasadena, California 91109

Dunbar, Carl Owen, 1944 (6), 1615 Santa Barbara Drive, Dunedin, Florida 33528

Dunn, Leslie Clarence, 1943 (8), 635 West 247th Street, New York, New York 10471

Dunning, John Ray, 1948 (3), School of Engineering and Applied Science, 510 Mudd Building, Columbia University, New York, New York 10027

du Vigneaud, Vincent, 1944 (14), Cornell University Medical College, 1300 York Avenue, New York, New York 10021

Dyson, Freeman John, 1964 (3), Institute for Advanced Study, Princeton, New Jersey 08540

Eagle, Harry, 1963 (10), Department of Cell Biology, Albert Einstein College of Medicine, Bronx, New York 10461

Eckart, Carl, 1953 (13), University of California, San Diego, La Jolla, California 92038

Edgerton, Harold Eugene, 1964 (4), Department of Electrical Engineering, Massachusetts Institute of Technology, Cambridge, Massachusetts 02139

Edsall, John Tileston, 1951 (14), The Biological Laboratories, Harvard University, 16 Divinity Avenue, Cambridge, Massachusetts 02138

Eggan, Fred Russell, 1963 (11), Department of Anthropology, University of Chicago, 1126 East 59th Street, Chicago, Illinois 60637

Eilenberg, Samuel, 1959 (1), Department of Mathematics, Columbia University, New York, New York 10027 
Eisenhart, Luther Pfahler, 1922 (1), 25 Alexander Street, Princeton, New Jersey 08541

Elderfield, Robert Cooley, 1949 (5), Department of Chemistry, University of Michigan, Ann Arbor, Michigan 48104

Elsasser, Walter Maurice, 1957 (13), Department of Geology, Princeton University, Princeton, New Jersey 08540

Emerson, Alfred Edwards, 1962 (8), Huletts Landing, New York 12841

Emmett, Paul Hugh, 1955 (5), Department of Chemistry, Johns Hopkins University, Baltimore, Maryland 21218

Enders, John Franklin, 1953 (10), The Children's Hospital Medical Center, 300 Longwood Avenue, Boston, Massachusetts 02115

Epstein, Paul Sophus, 1930 (3), 1484 Oakdale Street, Pasadena, California 91106

Erlanger, Joseph, 1922 (9), 5127 Waterman Boulevard, St. Louis, Missouri 63108

Esau, Katherine, 1957 (7), Department of Biological Sciences, University of California, Santa Barbara, California 93018

Estes, William Kaye, 1963 (12), Department of Psychology, Stanford University, Stanford, California 94305

Evans, Griffith Conrad, 1933 (1), Department of Mathematics, University of California, Berkeley, California 94720

Evans, Herbert McLean, 1927 (9), Institute of Experimental Biology, University of California, Berkeley, California 94720

Ewing, William Maurice, 1948 (13), Lamont Geological Observatory of Columbia University, Torrey Cliff, Palisades, New York 10964

Eyring, Henry, 1945 (5), Graduate School, University of Utah, Salt Lake City, Utah 84112

Fairbank, William Martin, 1963 (3), Department of Physics, Stanford University, Stanford, California 94305

Feller, William, 1960 (1), Fine Hall, Princeton University, Princeton, New Jersey 08540

Fenn, Wallace Osgood, 1943 (9), School of Medicine and Dentistry, University of Rochester, 260 Crittenden Boulevard, Rochester, New York 14620

Ferry, John Douglass, 1959 (5), Department of Chemistry, University of Wisconsin, Madison, Wisconsin 53706

Feynman, Richard Phillips, 1954 (3), Norman Bridge Laboratory of Physics, California Institute of Technology, Pasadena, California 91109

Fieser, Louis Frederick, 1940 (5), Harvard University, Cambridge, Massachusetts 02138

Fisk, James Brown, 1954 (4), Bell Telephone Laboratories, Incorporated, Murray Hill, New Jersey 07971

Fletcher, Harvey, 1935 (4), 293 Eyring Science Center, Brigham Young University, Provo, Utah 84601

Flexner, Louis Barkhouse, 1964 (8), School of Medicine, University of Pennsylvania, Philadelphia, Pennsylvania 19104

Flory, Paul John, 1953 (5), Department of Chemistry, Stanford University, Stanford, California 94305

Folkers, Karl August, 1948 (5), Stanford Research Institute, Menlo Park, California 94025

Foote, Paul Darwin, 1943 (4), 5144 Macomb Street, N. W., Washington, D. C. 20016

Forbes, Alexander, 1936 (9), 610 Harland Street, Milton, Massachusetts 02186 
Forbush, Scott Ellsworth, 1962 (13), Department of Terrestrial Magnetism, Carnegie Institution of Washington, 5241 Broad Branch Road, N. W., Washington, D. C. 20015

Fowler, William Alfred, 1956 (3), W. K. Kellogg Radiation Laboratory, California Institute of Technology, Pasadena, California 91109

Francis, Thomas, Jr., 1948 (10), Department of Epidemiology, School of Public Health, University of Michigan, Ann Arbor, Michigan 48104

Fred, Edwin Broun, 1931 (7), University of Wisconsin, Madison, Wisconsin 53706

French, Charles Stacy, 1963 (7), Department of Plant Biology, Carnegie Institution of Washington, Stanford, California 94305

Friedman, Herbert, 1960 (13), United States Naval Research Laboratory (Code 7100), Washington, D. C. 20390

Friedmann, Herbert, 1962 (8), Los Angeles County Museum, 900 Exposition Boulevard, Los Angeles, California 90007

Friedrichs, Kurt Otto, 1959 (1), Courant Institute of Mathematical Sciences, New York University, 25 Waverly Place, New York, New York 10003

Fruton, Joseph Stewart, 1952 (14), Department of Biochemistry, Yale University, 333 Cedar Street, New Haven, Connecticut 06520

Fuoss, Raymond Matthew, 1951 (5), 57 Mill Rock Road, New Haven, Connecticut 06511

Fuson, Reynold Clayton, 1944 (5), Department of Chemistry, University of Nevada, Reno, Nevada 89507

Galambos, Robert, 1960 (12), Yale University, 333 Cedar Street, New Haven, Connecticut 06520

Gamow, George, 1953 (3), Department of Physics, University of Colorado, Boulder, Colorado 80304

Garrels, Robert Minard, 1962 (6), 302 Hoffman Laboratory, Department of Geological Sciences, Harvard University, Cambridge, Massachusetts 02138

Gates, Marshall DeMotte, Jr., 1958 (5), Department of Chemistry, University of Rochester, Rochester, New York 14627

Gell-Mann, Murray, 1960 (3), Department of Physics, California Institute of Technology, Pasadena, California 91109

Gerard, Ralph Waldo, 1955 (9), University of California, Irvine, California 92650

Giauque, William Francis, 1936 (5), Department of Chemistry, University of California, Berkeley, California 94720

Gibbs, William Francis, 1949 (4), One Broadway, New York, New York 10004

Gilliland, Edwin Richard, 1948 (4), Room 12-117, Department of Chemical Engineering, Massachusetts Institute of Technology, Cambridge, Massachusetts 02139

Gilluly, James, 1947 (6), United States Geological Survey, Denver Federal Center, Denver, Colorado 80225

Gilman, Alfred, 1964 (9), Department of Pharmacology, Albert Einstein College of Medicine of Yeshiva University, New York, New York 10461

Gilman, Henry, 1945 (5), Department of Chemistry, Iowa State University, Ames, Iowa 50012

Glaser, Donald Arthur, 1962 (3), 229 Virus Laboratory, University of California, Berkeley, California 94720

Glass, Hiram Bentley, 1959 (8), Department of Biology, Johns Hopkins University, Baltimore, Maryland 21218 
Goddard, David Rockwell, 1950 (7), Provost's Office, 102 College Hall, University of Pennsylvania, Philadelphia, Pennsylvania 19104

Gödel, Kurt, 1955 (1), The Institute for Advanced Study, Princeton, New Jersey 08540

Goebel, Walther Frederick, 1958 (10), Rockefeller Institute, New York, New York 10021

Goldberg, Leo, 1958 (2), Harvard College Observatory, Cambridge, Massachusetts 02138

Goldberger, Marvin Leonard, 1963 (3), Palmer Physical Laboratory, Princeton University, Princeton, New Jersey 08540

Goldhaber, Maurice, 1958 (3), Director's Office, Brookhaven National Laboratory, Upton, Long Island, New York 11973

Gordy, Walter, 1964 (3), Department of Physics, Duke University, Durham, North Carolina 27706

Goudsmit, Samuel Abraham, 1947 (3), Department of Physics, Brookhaven National Laboratory, Upton, Long Island, New York 11973

Graham, Clarence Henry, 1946 (12), Department of Psychology, Columbia University, New York, New York 10027

Green, David Ezra, 1962 (14), Institute for Enzyme Research, University of Wisconsin, 1710 University Avenue, Madison, Wisconsin 53706

Greenewalt, Crawford Hallock, 1952 (4), E. I. du Pont de Nemours and Company, Incorporated, Wilmington, Delaware 19898

Greenstein, Jesse Leonard, 1957 (2), Mount Wilson and Palomar Observatories, California Institute of Technology, Pasadena, California 91106

Gregory, William King, 1927 (6), P. O. Box 35, Woodstock, New York 12498

Griffin, Donald Redfield, 1960 (8), The Biological Laboratories, Harvard University, 16 Divinity Avenue, Cambridge, Massachusetts 02138

Griggs, David Tressel, 1952 (13), Institute of Geophysics and Planetary Physics, University of California, Los Angeles, California 90024

Guilford, Joy Paul, 1954 (12), P. O. Box 1288, Beverly Hills, California 90213

Gunn, Ross, 1951 (13), 4437 Lowell Street, N. W., Washington, D. C. 20016

Gutowsky, Herbert Sander, 1960 (5), Department of Chemistry and Chemical Engineering, University of Illinois, Urbana, Illinois 61803

Hallowell, Alfred Irving, 1961 (11), Department of Anthropology, The University Museum, 33rd and Spruce Streets, Philadelphia, Pennsylvania 19104

Hamburger, Viktor, 1953 (8), Department of Zoology, Washington University, St. Louis, Missouri 63130

Hammett, Louis Plack, 1943 (5), Route 4, Box 310, Newton, New Jersey 07860

Hammond, George Simms, 1963 (5), Gates and Crellin Laboratories of Chemistry, California Institute of Technology, Pasadena, California 91109

Handler, Philip, 1964 (14), Department of Biochemistry, Duke University Medical Center, Durham, North Carolina 27706

Harlow, Harry F., 1951 (12), Primate Laboratory, 22 North Charter Street, Madison, Wisconsin 53715

Harned, Herbert Spencer, 1950 (5), Sterling Chemistry Laboratory, Yale University, 225 Prospect Street, New Haven, Connecticut 06520

Hartline, Haldan Keffer, 1948 (9), Rockefeller Institute, New York, New York 10021

Haskins, Caryl Parker, 1956 (8), Carnegie Institution of Washington, $1530 \mathrm{P}$ Street, N. W., Washington, D. C. 20005 
Hassid, William Zev, 1958 (14), Department of Biochemistry, Biochemistry Building, University of California, Berkeley, California 94720

Hastings, Albert Baird, 1939 (9), Scripps Clinic and Research Foundation, 476 Prospect Street, La Jolla, California 92037

Haurwitz, Bernhard, 1960 (13), National Center for Atmospheric Research, Boulder, Colorado 80304

Haury, Emil Walter, 1956 (11), Department of Anthropology, University of Arizona, Tucson, Arizona 85721

Hauser, Charles Roy, 1958 (5), Department of Chemistry, Duke University, Durham, North Carolina 27706

Hedberg, Hollis Dow, 1960 (6), Department of Geology, Princeton University, Princeton, New Jersey 08540

Heidelberger, Michael, 1942 (10), Department of Pathology, New York University School of Medicine, 550 First Avenue, New York, New York 10016

Hendricks, Sterling Brown, 1952 (7), Plant Industry Station, Beltsville, Maryland 20705

Herb, Raymond George, 1955 (3), Department of Physics, University of Wisconsin, Madison, Wisconsin 53706

Herbig, George Howard, 1964 (2), Lick Observatory, Mount Hamilton, California 95140

Herget, Paul, 1962 (2), Cincinnati Observatory, Observatory Place, Cincinnati, Ohio 45208

Hershey, Alfred Day, 1958 (7), Genetics Research Unit, Carnegie Institution of Washington, Cold Spring Harbor, Long Island, New York 11724

Herzfeld, Karl Ferdinand, 1960 (3), Department of Physics, Catholic University of America, Washington, D. C. 20017

Hess, Harry Hammond, 1952 (6), Department of Geology, Princeton University, Princeton, New Jersey 08540

Hewett, Donnel Foster, 1937 (6), 345 Middlefield Road, Menlo Park, California 94025

Hildebrand, Joel Henry, 1929 (5), Department of Chemistry, University of California, Berkeley, California 94720

Hilgard, Ernest Ropiequet, 1948 (12), Department of Psychology, Stanford University, Stanford, California 94305

Hille, Carl Einar, 1953 (1), 210 Edwards Street, New Haven, Connecticut 06511

Hirschfelder, Joseph Oakland, 1953 (5), Theoretical Chemistry Institute, University of Wisconsin, 1112 W. Johnson Street, Madison, Wisconsin 53706

Hisaw, Frederick Lee, 1947 (8), The Biological Laboratories, Harvard University, 16 Divinity Avenue, Cambridge, Massachusetts 02138

Hofmann, Klaus Heinrich, 1963 (14), Department of Biochemistry, University of Pittsburgh School of Medicine, Pittsburgh, Pennsylvania 15213

Hofstadter, Robert, 1958 (3), Department of Physics, Stanford University, Stanford, California 94305

Hollaender, Alexander, 1957 (7), Biology Division, Oak Ridge National Laboratory, P. O. Box Y, Oak Ridge, Tennessee 37831

Holtfreter, Johannes, 1955 (8), Biological Laboratories, University of Rochester, Rochester, New York 14627

Hoover, Herbert, 1922 (4), Waldorf Astoria Towers, New York, New York 10022

Horecker, Bernard Leonard, 1961 (14), Department of Molecular Biology, Albert Einstein College of Medicine of Yeshiva University, New York, New York 10461 
Hornig, Donald Frederick, 1957 (5), Executive Office Building, Washington, D. C. 20506

Horsfall, Frank Lappin, Jr., 1948 (10), Sloan-Kettering Institute for Cancer Research, New York, New York 10021

Horsfall, James Gordon, 1953 (7), Connecticut Agricultural Experiment Station, Box 1106, New Haven, Connecticut 06504

Hotchkiss, Rollin Douglas, 1961 (10), Rockefeller Institute, New York, New York 10021

Hottel, Hoyt Clarke, 1963 (4), Room 12-110, Department of Chemical Engineering, Massachusetts Institute of Technology, Cambridge, Massachusetts 02139

Houston, William Vermillion, 1943 (3), Rice University, Houston, Texas 77001

Hubbert, Marion King, 1955 (6), U. S. Geological Survey, Department of the Interior, Washington, D. C. 20242

Hubbs, Carl Leavitt, 1952 (8), Scripps Institution of Oceanography, La Jolla, California 92038

Huebner, Robert Joseph, 1960 (10), National Institutes of Health, Bethesda, Maryland 20014

Huggins, Charles Brenton, 1949 (10), The Ben May Laboratory for Cancer Research, University of Chicago, 950 East 59th Street, Chicago, Illinois 60637

Hull, Albert Wallace, 1929 (3), General Electric Research Laboratory, The Knolls, Schenectady, New York 12301

Hunsaker, Jerome Clark, 1935 (4), Room 33-207, Massachusetts Institute of Technology, Cambridge, Massachusetts 02139

Hutchinson, George Evelyn, 1950 (8), Osborn Memorial Laboratories, Yale University, New Haven, Connecticut 06520

Hutchison, Clyde Allen, Jr., 1963 (5), The Enrico Fermi Institute for Nuclear Studies, University of Chicago, Chicago, Illinois 60637

Hyman, Libbie Henrietta, 1961 (8), American Museum of Natural History, Central Park West at 79th Street, New York, New York 10024

Inghram, Mark Gordon, 1961 (3), Department of Physics, University of Chicago, Chicago, Illinois 60637

Ingle, Dwight Joyce, 1963 (9), Department of Physiology, University of Chicago, 951 East 58th Street, Chicago, Illinois 60637

Irwin, Malcolm Robert, 1950 (8), Department of Genetics, University of Wisconsin, Madison, Wisconsin 53706

Iselin, Columbus O'Donnell, 1951 (13), Woods Hole Oceanographic Institution, Woods Hole, Massachusetts 02543

Jacobs, Merkel Henry, 1939 (8), School of Medicine, University of Pennsylvania, Philadelphia, Pennsylvania 19104

Jacobs, Walter Abraham, 1932 (5), Rockefeller Institute, New York, New York 10021

Jacobson, Nathan, 1954 (1), Department of Mathematics, Yale University, New Haven, Connecticut 06520

James, Harold Lloyd, 1962 (6), Department of Geology, University of Minnesota, Minneapolis, Minnesota 55455

Jeffries, Zay, 1939 (4), General Electric Company, 1 Plastics Avenue, Pittsfield, Massachusetts 01201

John, Fritz, 1964 (1), Courant Institute of Mathematical Sciences, New York University, 4 Washington Place, New York, New York 10003

Johnson, John Raven, 1948 (5), Department of Chemistry, Cornell University, Ithaca, New York 14850 
Johnson, William Summer, 1952 (5), Department of Chemistry, Stanford University, Stanford, California 94305

Joy, Alfred Harrison, 1944 (2), Mount Wilson and Palomar Observatories, 813 Santa Barbara Street, Pasadena, California 91106

Kalckar, Herman Moritz, 1959 (14), Biochemical Research Department, Massachusetts General Hospital, Boston, Massachusetts 02114

Kamen, Martin David, 1962 (14), Department of Chemistry, The First College, University of California, San Diego, La Jolla, California 92038

Kaplan, Joseph, 1957 (13), Department of Physics, University of California, Los Angeles, California 90024

Kaufmann, Berwind Petersen, 1952 (7), Department of Zoology, University of Michigan, Ann Arbor, Michigan 48104

Kauzmann, Walter Joseph, 1964 (5), Department of Chemistry, Princeton University, Princeton, New Jersey 08540

Kellogg, Arthur Remington, 1951 (8), 5305 28th Street, N. W., Washington, D. C. 20015

Kelly, Mervin J., 1945 (4), 2 Windemere Terrace, Short Hills, New Jersey 07078

Kemble, Edwin Crawford, 1931 (3), Physics Laboratories, Harvard University, Cambridge, Massachusetts 02138

Kendall, Edward Calvin, 1950 (14), 3 Queenston Place, Princeton, New Jersey 08540

Kennedy, Eugene Patrick, 1964 (14), Department of Biological Chemistry, Harvard Medical School, 25 Shattuck Street, Boston, Massachusetts 02115

Kerst, Donald William, 1951 (3), Physics Department, Sterling Hall, University of Wisconsin, Madison, Wisconsin 53706

Kety, Seymour Solomon, 1962 (9), Laboratory of Clinical Science, National Institute of Mental Health, National Institutes of Health, Bethesda, Maryland 20014

Keyes, Frederick George, 1930 (5), Massachusetts Institute of Technology, Cambridge, Massachusetts 02139

Kimball, George Elbert, 1954 (5), Arthur D. Little, Inc., 35 Acorn Park, Cambridge, Massachusetts 02140

King, Charles Glen, 1951 (14), Institute of Nutrition Sciences, Columbia University, 562 West 168th Street, New York, New York 10032

Kinzel, Augustus Braun, 1960 (4), Union Carbide Corporation, 270 Park Avenue, New York, New York 10017

Kistiakowsky, George Bogdan, 1939 (5), Department of Chemistry, Harvard University, 12 Oxford Street, Cambridge, Massachusetts 02138

Kittel, Charles, 1957 (3), Department of Physics, University of California, Berkeley, California 94720

Klïver, Heinrich, 1957 (12), Culver Hall, University of Chicago, Chicago, Illinois 60637

Knopf, Adolph, 1931 (6), Department of Geology, Stanford University, Stanford, California 94305

Knopoff, Leon, 1963 (13), Institute of Geophysics and Planetary Physics, University of California, Los Angeles, California 90024

Köhler, Wolfgang, 1947 (12), P. O. Box 32, Lebanon, New Hampshire 03766

Kolthoff, Izaak Maurits, 1958 (5), School of Chemistry, University of Minnesota, Minneapolis, Minnesota 55455

Kornberg, Arthur, 1957 (14), Department of Biochemistry, Stanford University Medical School, Palo Alto, California 94304 
Kramer, Paul Jackson, 1962 (7), Department of Botany, Duke University, Durham, North Carolina 27706

Kraus, Charles August, 1925 (5), 92 Keene Street, Providence, Rhode Island 02906

Krauskopf, Konrad Bates, 1959 (6), School of Earth Sciences, Stanford University, Stanford, California 94305

Krayer, Otto, 1964 (9), Department of Pharmacology, Harvard Medical School, 25 Shattuck Street, Boston, Massachusetts 02115

Kuffler, Stephen William, 1964 (9), Department of Pharmacology, Harvard Medical School, 25 Shattuck Street, Boston, Massachusetts 02115

Kuiper, Gerard Peter, 1950 (2), Lunar and Planetary Laboratory, University of Arizona, Tucson, Arizona 85721

Kusch, Polykarp, 1956 (3), Department of Physics, Columbia University, New York, New York 10027

Lamb, Willis Eugene, Jr., 1954 (3), Sloane Physics Laboratory, Yale University, New Haven, Connecticut 06520

Lambert, Walter Davis, 1949 (13), P. O. Box 1025, Canaan, Connecticut 06018

La Mer, Victor Kuhn, 1945 (5), 353 Moore Avenue, Leonia, New Jersey 07605

Land, Edwin Herbert, 1953 (3), Polaroid Corporation, Cambridge, Massachusetts 02139

Landis, Eugene Markley, 1954 (9), Department of Physiology, Harvard Medical School, 25 Shattuck Street, Boston, Massachusetts 02115

Lardy, Henry Arnold, 1958 (14), Institute for Enzyme Research, University of Wisconsin, 1702 University Avenue, Madison, Wisconsin 53705

Lauritsen, Charles Christian, 1941 (3), W. K. Kellogg Radiation Laboratory, California Institute of Technology, Pasadena, California 91109

Lederberg, Joshua, 1957 (14), Genetics Department, School of Medicine, Stanford University, Palo Alto, California 94304

Lee, Tsung-Dao, 1964 (3), Department of Physics, Columbia University, New York, New York 10027

Lefschetz, Solomon, 1925 (1), Fine Hall, Princeton University, Princeton, New Jersey 08540

Lehninger, Albert Lester, 1956 (14), School of Medicine, Johns Hopkins University, 725 North Wolfe Street, Baltimore, Maryland 21205

Leonard, Nelson Jordan, 1955 (5), Department of Chemistry and Chemical Engineering, University of Illinois, Urbana, Illinois 61803

Lerner, I. Michael, 1959 (8), Genetics Department, Mulford Hall, University of California, Berkeley, California 94720

Lewis, Warren Harmon, 1936 (8), The Wistar Institute of Anatomy and Biology, Philadelphia, Pennsylvania 19104

Lewis, Warren Kendall, 1938 (4), Room 12-112, Massachusetts Institute of Technology, Cambridge, Massachusetts 02139

Lewy, Hans, 1964 (1), Department of Mathematics, University of California, Berkeley, California 94720

Libby, Willard Frank, 1950 (5), Department of Chemistry, University of California, 405 Hilgard Avenue, Los Angeles, California 90024

Lin, Chia-Chiao, 1962 (1), Department of Mathematics, Massachusetts Institute of Technology, Cambridge, Massachusetts 02139

Lind, Samuel Colville, 1930 (5), P. O. Box P, Oak Ridge, Tennessee 37831

Lindsley, Donald Benjamin, 1952 (12), Department of Psychology, University of California, Los Angeles, California 90024 
Link, Karl Paul, 1946 (14), Department of Biochemistry, University of Wisconsin, Madison, Wisconsin 53706

Lipmann, Fritz Albert, 1950 (14), Rockefeller Institute, New York, New York 10021

Lipscomb, William Nunn, 1961 (5), Department of Chemistry, Harvard University, 12 Oxford Street, Cambridge, Massachusetts 02138

Little, Clarence Cook, 1945 (10), R. F. D. 1, Ellsworth, Maine 04605

Lloyd, David Pierce Caradoc, 1953 (9), Rockefeller Institute, New York, New York 10021

Loeb, Robert Frederick, 1946 (9), 950 Park Avenue, New York, New York 10028

Long, Cyril Norman Hugh, 1948 (9), Yale University School of Medicine, 333 Cedar Street, New Haven, Connecticut 06510

Long, Esmond Ray, 1946 (10), Pedlar Mills, Virginia 24583

Long, Franklin A., 1962 (5), Department of Chemistry, Cornell University, Ithaca, New York 14850

Longsworth, Lewis Gibson, 1947 (5), Rockefeller Institute, New York, New York 10021

Longwell, Chester Ray, 1935 (6), 1820 Mark Twain Street, Palo Alto, California 94304

Loomis, Alfred Lee, 1941 (4), The Loomis Institute for Scientific Research, Incorporated, Room 2420, 14 Wall Street, New York, New York 10005

Loomis, Francis Wheeler, 1949 (3), 804 West Illinois Street, Urbana, Illinois 61801

Lorente de Nó, Rafael, 1950 (9), Rockefeller Institute, New York, New York 10021

Lothrop, Samuel Kirkland, 1951 (11), Peabody Museum, Harvard University, Cambridge, Massachusetts 02138

Lovering, Thomas Seward, 1949 (6), Geologic Division, United States Geological Survey, Denver Federal Center, Denver, Colorado 80225

Lowry, Oliver Howe, 1964 (14), School of Medicine, Washington University, Euclid Avenue and Kingshighway, St. Louis, Missouri 63110

Luria, Salvador Edward, 1960 (10), Department of Biology, Massachusetts Institute of Technology, Cambridge, Massachusetts 02139

MacDonald, Gordon James Fraser, 1962 (13), Institute of Geophysics and Planetary Physics, University of California, Los Angeles, California 90024

MacInnes, Duncan Arthur, 1937 (5), Rockefeller Institute, New York, New York 10021

Mackey, George Whitelaw, 1962 (1), Department of Mathematics, Harvard University, Cambridge, Massachusetts 02138

Mackin, Joseph Hoover, 1963 (6), Department of Geology, University of Texas, Austin, Texas 78712

Mac Lane, Saunders, 1949 (1), Eckhart Hall, University of Chicago, Chicago, Illinois 60637

MacLeod, Colin Munro, 1955 (10), Office of Science and Technology, Room 200, Executive Office Building, Washington, D. C. 20506

Magoun, Horace Winchell, 1955 (9), Graduate Division, University of California, Los Angeles, California 90024

Mangelsdorf, Paul Christoph, 1945 (7), Botanical Museum, Harvard University, Cambridge, Massachusetts 02138

Mark, Herman Francis, 1961 (5), Polymer Research Institute, Polytechnic Institute of Brooklyn, Brooklyn, New York 1.1201 
Marshak, Robert Eugene, 1958 (3), Department of Physics and Astronomy, University of Rochester, Rochester, New York 14627

Marshall, Eli Kennerly, Jr., 1943 (9), Department of Medicine, Johns Hopkins Hospital, Baltimore, Maryland 21205

Marvel, Carl Shipp, 1938 (5), Department of Chemistry, University of Arizona, Tucson, Arizona 85721

Maxcy, Kenneth Fuller, 1950 (10), The Park Lynn Apartments, 4 Upland Road, Baltimore, Maryland 21210

Mayall, Nicholas Ulrich, 1949 (2), Kitt Peak National Observatory, 950 North Cherry Avenue, Box 4130, Tucson, Arizona 85719

Mayer, Joseph Edward, 1946 (5), The First College, University of California, San Diego, La Jolla, California 92038

Mayer, Maria Goeppert, 1956 (3), School of Science and Engineering, University of California, San Diego, La Jolla, California 92038

Maynard, Leonard Amby, 1944 (14), Savage Hall, Cornell University, Ithaca, New York 14850

Mayr, Ernst, 1954 (8), Museum of Comparative Zoology at Harvard College, Oxford Street, Cambridge, Massachusetts 02138

Mazia, Daniel, 1960 (8), Department of Zoology, University of California, Berkeley, California 94720

McCarty, Maclyn, 1963 (10), Rockefeller Institute, New York, New York 10021

McClintock, Barbara, 1944 (7), Carnegie Institution of Washington, Cold Spring Harbor, Long Island, New York 11724

McCollum, Elmer Verner, 1920 (14), Gilman Hall, Johns Hopkins University, Baltimore, Maryland 21218

McElroy, William David, 1963 (14), Department of Biology, Johns Hopkins University, Baltimore, Maryland 21218

McElvain, Samuel Marion, 1949 (5), Department of Chemistry, University of Wisconsin, Madison, Wisconsin 53706

McMaster, Philip Duryeé, 1952 (10), Rockefeller Institute, New York, New York 10021

McMillan, Edwin Mattison, 1947 (3), Lawrence Radiation Laboratory, University of California, Berkeley, California 94720

McShane, Edward James, 1948 (1), Department of Mathematics, University of Virginia, Charlottesville, Virginia 22903

Meggers, William Frederick, 1954 (3), 2904 Brandywine Street, N. W., Washington, D. C. 20008

Mehl, Robert Franklin, 1958 (4), Beethovenstrasse 32, Zurich 2, Switzerland

Menzel, Donald Howard, 1948 (2), Harvard College Observatory, Cambridge, Massachusetts 02138

Metz, Charles William, 1948 (8), P. O. Box 714, Woods Hole, Massachusetts 02543

Meyer, Karl Friederich, 1940 (10), George Williams Hooper Foundation, University of California Medical Center, San Francisco, California 94122

Miles, Walter Richard, 1933 (12), Box 100, Naval Medical Research Laboratory, U. S. N. Submarine Base, Groton, Connecticut 06342

Miller, Alden Holmes, 1957 (8), Museum of Vertebrate Zoology, University of California, Berkeley, California 94720

Miller, Charles Phillip, 1956 (10), Department of Medicine, University of Chicago, Chicago, Illinois 60637

Miller, George Armitage, 1962 (12), Center for Cognitive Studies, Harvard University, 61 Kirkland Street, Cambridge, Massachusetts 02138 
Miller, Neal Elgar, 1958 (12), Department of Psychology, Yale University, 333 Cedar Street, New Haven, Connecticut 06520

Millikan, Clark Blanchard, 1964 (4), Graduate Aeronautical Laboratories, California Institute of Technology, Pasadena, California 91109

Milnor, John Willard, 1963 (1), Fine Hall, Princeton University, P. O. Box 708, Princeton, New Jersey 08540

Minkowski, Rudolph Leo, 1959 (2), Radio Astronomy Laboratory, University of California, Berkeley, California 94720

Mirsky, Alfred Ezra, 1954 (8), Rockefeller Institute, New York, New York 10021

Montgomery, Deane, 1955 (1), School of Mathematics, The Institute for Advanced Study, Princeton, New Jersey 08540

Moore, John Alexander, 1963 (8), Department of Zoology, Columbia University, New York, New York 10027

Moore, Robert Lee, 1931 (1), University of Texas, Austin, Texas 78712

Moore, Stanford, 1960 (14), Rockefeller Institute, New York, New York 10021

Morgan, William Wilson, 1956 (2), Yerkes Observatory, University of Chicago, Williams Bay, Wisconsin 53191

Morrey, Charles Bradfield, Jr., 1962 (1), Department of Mathematics, University of California, Berkeley, California 94720

Morse, Harold Marston, 1932 (1), The Institute for Advanced Study, Princeton, New Jersey 08540

Morse, Philip McCord, 1955 (3), Department of Physics, Massachusetts Institute of Technology, Cambridge, Massachusetts 02139

Movius, Hallam Leonard, Jr., 1957 (11), Peabody Museum, Harvard University, Cambridge, Massachusetts 02138

Muller, Hermann Joseph, 1931 (8), City of Hope Medical Center, Duarte, California 91010

Mulliken, Robert Sanderson, 1936 (3), Laboratory of Molecular Structure and Spectra, Department of Physics, University of Chicago, Chicago, Illinois 60637

Munk, Walter Heinrich, 1956 (13), La Jolla Laboratories, Institute of Geophysics and Planetary Physics, University of California, San Diego, La Jolla, California 92038

Murdock, George Peter, 1964 (11), Department of Anthropology, University of Pittsburgh, Pittsburgh, Pennsylvania 15213

Murnaghan, Francis Dominic, 1942 (1), 6202 Sycamore Road, Baltimore, Maryland 21212

Neel, James Van Gundia, 1963 (8), Department of Human Genetics, University of Michigan Medical School, Ann Arbor, Michigan 48104

Neff, William Duwayne, 1964 (12), Center for Brain Research, University of Rochester, Rochester, New York 14627

Neurath, Hans, 1961 (14), Department of Biochemistry, University of Washington, Seattle, Washington 98105

Newman, Melvin Spencer, 1956 (5), 2239 Onandaga Drive, Columbus, Ohio 43221

Neyman, Jerzy, 1963 (1), Department of Statistics, University of California, Berkeley, California 94720

Nier, Alfred Otto C., 1950 (3), School of Physics, University of Minnesota, Minneapolis, Minnesota 55455

Nolan, Thomas Brennan, 1951 (6), 2219 California Street, N. W., Washington, D. C. 20008 
Northrop, John Howard, 1934 (14), Department of Bacteriology, University of California, Berkeley, California 94720

Noyes, William Albert, Jr., 1943 (5), Department of Chemistry, University of Texas, Austin, Texas 78712

O'Brien, Brian, 1954 (3), Box 117, Pomfret, Connecticut 06258

Ochoa, Severo, 1957 (14), New York University School of Medicine, 550 First Avenue, New York, New York 10016

Olson, Harry Ferdinand, 1959 (4), RCA Laboratories, David Sarnoff Research Center, Princeton, New Jersey 08541

Oncley, John Lawrence, 1947 (14), Biophysics Research Division, Institute of Science and Technology, University of Michigan, Ann Arbor, Michigan 48104

Onsager, Lars, 1947 (5), Sterling Chemistry Laboratory, Yale University, New Haven, Connecticut 06520

Opie, Eugene Lindsay, 1923 (10), Rockefeller Institute, New York, New York 10021

Oppenheimer, J. Robert, 1941 (3), The Institute for Advanced Study, Princeton, New Jersey 08540

Painter, Theophilus Shickel, 1938 (8), University of Texas, Austin, Texas 78712

Pais, Abraham, 1962 (3), Rockefeller Institute, New York, New York 10021

Palade, George Emil, 1961 (8), Rockefeller Institute, New York, New York 10021

Panofsky, Wolfgang K. H., 1954 (3), Stanford Linear Accelerator Center, Stanford University, Stanford, California 94305

Patterson, Bryan, 1963 (6), Museum of Comparative Zoology at Harvard College, Oxford Street, Cambridge, Massachusetts 02138

Paul, John Rodman, 1945 (10), Yale University School of Medicine, 333 Cedar Street, New Haven, Connecticut 06510

Pauling, Linus, 1933 (5), Big Sur, California 93920

Pekeris, Chaim Leib, 1952 (13), Department of Applied Mathematics, The Weizmann Institute of Science, Rehovot, Israel

Perlman, Isadore, 1963 (5), Department of Nuclear Chemistry, Lawrence Radiation Laboratory, Berkeley, California 94720

Pfaffmann, Carl, 1959 (12), Walter S. Hunter Laboratory of Psychology, Brown University, Providence, Rhode Island 02912

Pickering, William Hayward, 1962 (4), Jet Propulsion Laboratory, 4800 Oak Grove Drive, Pasadena, California 91103

Pierce, John Robinson, 1955 (4), Bell Telephone Laboratories, Incorporated, Murray Hill, New Jersey 07971

Piggot, Charles Snowden, 1946 (13), Holly Grove, Route 2, Box 68, Lexington Park, Maryland 20653

Piore, Emanuel Ruben, 1963 (4), International Business Machines Corporation, 590 Madison Avenue, New York, New York 10022

Pittendrigh, Colin Stephenson, 1963 (8), Department of Biology, Princeton University, P. O. Box 704, Princeton, New Jersey 08540

Pitts, Robert Franklin, 1956 (9), Cornell University Medical College, 1300 York Avenue, New York, New York 10021

Pitzer, Kenneth Sanborn, 1949 (5), Rice University, Houston, Texas 77001

Porter, Keith Roberts, 1964 (8), The Biological Laboratories, Harvard University, 16 Divinity Avenue, Cambridge, Massachusetts 02138

Pound, Robert Vivian, 1961 (3), Lyman Laboratory of Physics, Harvard University, Cambridge, Massachusetts 02138 
Press, Frank, 1958 (13), Seismological Laboratory, California Institute of Technology, 220 North San Rafael Avenue, Pasadena, California 91105

Puck, Theodore Thomas, 1960 (10), Department of Biophysics, University of Colorado Medical Center, Denver, Colorado 80220

Purcell, Edward Mills, 1951 (3), Lyman Laboratory of Physics, Harvard University, Cambridge, Massachusetts 02138

Rabi, Isidor Isaac, 1940 (3), Department of Physics, Columbia University, New York, New York 10027

Ramsey, Norman Foster, 1952 (3), Lyman Laboratory of Physics, Harvard University, Cambridge, Massachusetts 02138

Raper, John Robert, 1964 (7), The Biological Laboratories, Harvard University, 16 Divinity Avenue, Cambridge, Massachusetts 02138

Raper, Kenneth Bryan, 1949 (7), Department of Bacterioloxy, University of Wisconsin, Madison, Wisconsin 53706

Raymond, Arthur Emmons, 1950 (4), 73 Oakmont Drive, Los Angeles, California 90049

Redfield, Alfred Clarence, 1958 (13), P. O. Box 106, Woods Hole, Massachusetts 02543

Reichelderfer, Francis Wilton, 1945 (13), 3031 Sedgwick Street, N. W., Washington, D. C. 20008

Revelle, Roger Randall, 1957 (13), 714 University Hall, University of California, Berkeley, California 94720

Rhoades, Marcus Morton, 1946 (7), Department of Botany, Indiana University, Bloomington, Indiana 47405

Rice, Oscar Knefler, 1964 (5), Department of Chemistry, University of North Carolina, Chapel Hill, North Carolina 27515

Rich, Arnold Rice, 1954 (10), Johns Hopkins Hospital, Baltimore, Maryland 21205

Richards, Alfred Newton, 1927 (9), 737 Rugby Road, Bryn Mawr, Pennsylvania 19010

Richards, Dickinson W., 1958 (9), Columbia University College of Physicians and Surgeons, 630 West 168th Street, New York, New York 10032

Richter, Curt Paul, 1948 (12), Johns Hopkins Hospital, Baltimore, Maryland 21205

Riddle, Oscar, 1939 (8), Route 4, Plant City, Florida 33566

Riggs, Lorrin Andrews, 1961 (12), Hunter Laboratory of Psychology, Brown University, Providence, Rhode Island 02912

Riker, Albert Joyce, 1951 (7), Department of Plant Pathology, University of Wisconsin, Madison, Wisconsin 53706

Rittenberg, David, 1953 (14), Columbia University College of Physicians and Surgeons, 630 West 168th Street, New York, New York 10032

Robbins, William Jacob, 1940 (7), 301 East 66th Street, New York, New York 10021

Roberts, John D., 1956 (5), Gates and Crellin Laboratories of Chemistry, California Institute of Technology, Pasadena, California 91109

Roberts, Richard Brooke, 1961 (7), Department of Terrestrial Magnetism, Carnegie Institution of Washington, 5241 Broad Branch Road, N. W., Washington, D. C. 20015

Robertson, Oswald Hope, 1943 (10), 9150 Los Gatos Highway, Santa Cruz, California 95062

Roeder, Kenneth David, 1964 (8), Department of Biology, Tufts University, Medford, Massachusetts 02155 
Romer, Alfred Sherwood, 1944 (8), Museum of Comparative Zoology, Harvard University, Oxford Street, Cambridge, Massachusetts 02138

Rose, William Cumming, 1936 (14), University of Illinois, Urbana, Illinois 61803

Rossi, Bruno Benedetto, 1950 (3), Room 26-569, Department of Physics, Massachusetts Institute of Technology, Cambridge, Massachusetts 02139

Rossini, Frederick Dominic, 1951 (5), University of Notre Dame, Notre Dame, Indiana 46556

Rous, Francis Peyton, 1927 (10), Rockefeller Institute, New York, New York 10021

Rouse, Irving, 1962 (11), Department of Anthropology, Yale University, Box 2114, Yale Station, New Haven, Connecticut 06520

Rubey, William Walden, 1945 (6), Department of Geology and Institute of Geophysics, University of California, Los Angeles, California 90024

Russell, Richard Joel, 1959 (6), Coastal Studies Institute, Louisiana State University, Baton Rouge, Louisiana 70803

Sabin, Albert Bruce, 1951 (10), The Children's Hospital Research Foundation, University of Cincinnati College of Medicine, Elland Avenue and Bethesda, Cincinnati, Ohio 45229

Sandage, Allan Rex, 1963 (2), Mount Wilson and Palomar Observatories, 813 Santa Barbara Street, Pasadena, California 91106

Savage, John Lucian, 1949 (4), 1651 Dahlia Street, Denver, Colorado 80220

Sax, Karl, 1941 (7), Bishop Hollow Road, Media, Pennsylvania 19064

Scatchard, George, 1946 (5), Department of Chemistry, Massachusetts Institute of Technology, Cambridge, Massachusetts 02139

Schairer, John Frank, 1953 (6), Geophysical Laboratory, Carnegie Institution of Washington, 2801 Upton Street, N. W., Washington, D. C. 20008

Schiff, Leonard Isaac, 1957 (3), Department of Physics, Stanford University, Stanford, California 94305

Schmidt, Carl Frederic, 1949 (9), Aviation Medical Acceleration Laboratory, United States Naval Air Development Center, Johnsville, Pennsylvania 18974

Schmidt-Nielsen, Knut, 1963 (8), Department of Zoology, Duke University, Durham, North Carolina 27706

Schmitt, Francis Otto, 1948 (8), Room 16-512, Department of Biology, Massachusetts Institute of Technology, Cambridge, Massachusetts 02139

Scholander, Per Fredrik, 1961 (8), Scripps Institution of Oceanography, University of California, La Jolla, California 92038

Schwarzschild, Martin, 1956 (2), Princeton University Observatory, 265 FitzRandolph Road, Princeton, New Jersey 08541

Schwinger, Julian, 1949 (3), Department of Physics, Harvard University, Cambridge, Massachusetts 02138

Seaborg, Glenn Theodore, 1948 (5), United States Atomic Energy Commission, Washington, D. C. 20545

Seares, Frederick Hanley, 1919 (2), Halekulani Hotel, Honolulu, Hawaii 96815

Sears, Ernest Robert, 1964 (7), U. S. Department of Agriculture, 108 Curtis Hall, University of Missouri, Columbia, Missouri 65202

Segrè, Emilio, 1952 (3), Department of Physics, University of California, Berkeley, California 94720

Seitz, Frederick, 1951 (3), Graduate School, University of Illinois, Urbana, Illinois 61803

Serber, Robert, 1952 (3), Department of Physics, Columbia University, New York, New York 10027 
Shane, Charles Donald, 1961 (2), P. O. Box 582, Santa Cruz, California 95061

Shannon, Claude Elwood, 1956 (1), Research Laboratory of Electronics, Massachusetts Institute of Technology, Cambridge, Massachusetts 02139

Shapiro, Harry Lionel, 1949 (11), American Museum of Natural History, Central Park West at 79th Street, New York, New York 10024

Shapley, Harlow, 1924 (2), Sharon Cross Road, Peterboro, New Hampshire 03458

Shedlovsky, Theodore, 1953 (5), Rockefeller Institute, New York, New York 10021

Sheehan, John Clark, 1957 (5), Department of Chemistry, Massachusetts Institute of Technology, Cambridge, Massachusetts 02139

Shemin, David, 1958 (14), Columbia University College of Physicians and Surgeons, 630 West 168th Street, New York, New York 10032

Sherwood, Thomas Kilgore, 1958 (4), Room 12-186, Department of Chemical Engineering, Massachusetts Institute of Technology, Cambridge, Massachusetts 02139

Shockley, William, 1951 (4), Stanford Electronics Laboratory, Stanford University, Stanford, California 94305

Shope, Richard Edwin, 1940 (10), Rockefeller Institute, New York, New York 10021

Simpson, George Gaylord, 1941 (6), Museum of Comparative Zoology at Harvard College, Cambridge, Massachusetts 02138

Simpson, John Alexander, 1959 (3), The Enrico Fermi Institute for Nuclear Studies, University of Chicago, Chicago, Illinois 60637

Sinnott, Edmund Ware, 1936 (7), Osborn Biological Laboratory, Yale University New Haven, Connecticut 06520

Skinner, Burrhus Frederic, 1950 (12), Memorial Hall, Harvard University, Cambridge, Massachusetts 02138

Skoog, Folke Karl, 1956 (7), Department of Botany, Birge Hall, University of Wisconsin, Madison, Wisconsin 53706

Slater, John Clarke, 1932 (3), Massachusetts Institute of Technology, Cambridge, Massachusetts 02139

Slepian, Joseph, 1941 (4), 1115 Lancaster Street, Pittsburgh, Pennsylvania 15218

Slichter, Louis Byrne, 1944 (13), Institute of Geophysics, University of California, Los Angeles, California 90024

Slipher, Vesto Melvin, 1921 (2), P. O. Box 326, Flagstaff, Arizona 86002

Smith, Albert Charles, 1963 (7), University of Hawaii, Honolulu, Hawaii 96822

Smith, Cyril Stanley, 1957 (4), Room 14N-317, Massachusetts Institute of Technology, Cambridge, Massachusetts 02139

Smith, Emil L., 1962 (14), Department of Biological Chemistry, University of California Medical Center, Los Angeles, California 90024

Smith, Lee Irvin, 1944 (5), School of Chemistry, University of Minnesota, Minneapolis, Minnesota 55455

Smith, Paul Althaus, 1947 (1), Department of Mathematics, Columbia University, New York, New York 10027

Smith, Philip Edward, 1939 (8), P. O. Box 6087, Carmel Valley Manor, Carmel, California 93921

Smyth, Charles Phelps, 1955 (5), Frick Chemical Laboratory, Princeton University, Princeton, New Jersey 08540

Snell, Esmond Emerson, 1955 (14), Department of Biochemistry, University of California, Berkeley, California 94720 
Soderberg, Carl Richard, 1947 (4), Room 1-207, Massachusetts Institute of Technology, Cambridge, Massachusetts 02139

Sonneborn, Tracy Morton, 1946 (8), 220 Jordan Hall, Indiana University, Bloomington, Indiana 47405

Spedding, Frank Harold, 1952 (5), Box 1129, ISU Station, Institute for Atomic Research, Iowa State University, Ames, Iowa 50012

Spence, Kenneth Wartinbe, 1955 (12), Department of Psychology, State University of Iowa, Iowa City, Iowa 52241

Spencer, Donald Clayton, 1961 (1), Department of Mathematics, Stanford University, Stanford, California 94305

Sperry, Roger Wolcott, 1960 (12), Division of Biology, California Institute of Technology, Pasadena, California 91109

Spitzer, Lyman, Jr., 1952 (2), Princeton University Observatory, 265 FitzRandolph Road, Princeton, New Jersey 08541

Sporn, Philip, 1962 (4), American Electric Power Company, Inc., 2 Broadway, New York, New York 10008

Stakman, Elvin Charles, 1934 (7), Institute of Agriculture, University of Minnesota, St. Paul, Minnesota 55101

Stanley, Wendell Meredith, 1941 (14), Virus Laboratory, University of California, Berkeley, California 94720

Stebbins, George Ledyard, 1952 (7), Department of Genetics, University of California, Davis, California 95616

Stebbins, Joel, 1920 (2), 69 University Drive, Menlo Park, California 94026

Steenrod, Norman Earl, 1956 (1), Fine Hall, Princeton University, P. O. Box 708, Princeton, New Jersey 08540

Stein, William Howard, 1960 (14), Rockefeller Institute, New York, New York 10021

Stern, Curt, 1948 (8), Department of Zoology, University of California, Berkeley, California 94720

Stern, Otto, 1945 (3), 759 Cragmont Avenue, Berkeley, California 94708

Stevens, Stanley Smith, 1946 (12), Memorial Hall, Harvard University, Cambridge, Massachusetts 02138

Steward, Julian H., 1954 (11), Department of Anthropology, 137 Davenport Hall, University of Illinois, Urbana, Illinois 61803

Stewart, Thomas Dale, 1962 (11), Museum of Natural History, Smithsonian Institution, Washington, D. C. 20560

Stockmayer, Walter Hugo, 1956 (5), Department of Chemistry, Dartmouth College, Hanover, New Hampshire 03755

Stoker, James Johnston, 1963 (1), New York University, 4 Washington Place, New York, New York 10003

Stommel, Henry Melson, 1961 (13), Massachusetts Institute of Technology, Cambridge, Massachusetts 02139

Stone, Marshall Harvey, 1938 (1), 303 Eckhart Hall, University of Chicago, Chicago, Illinois 60637

Stone, Wilson Stuart, 1960 (8), Genetics Foundation, University of Texas, Austin, Texas 78712

Stork, Gilbert Josse, 1960 (5), Department of Chemistry, Columbia University, New York, New York 10027

Stratton, Julius Adams, 1950 (4), Massachusetts Institute of Technology, Cambridge, Massachusetts 02139 
Straus, William Louis, Jr., 1962 (8), Department of Anatomy, School of Medicine, Johns Hopkins University, 725 North Wolfe Street, Baltimore, Maryland 21205

Street, Jabez Curry, 1953 (3), Jefferson Physical Laboratory, Harvard University, Cambridge, Massachusetts 02138

Sturtevant, Alfred Henry, 1930 (8), California Institute of Technology, Pasadena, California 91109

Suits, Chauncey Guy, 1946 (4), General Electric Research Laboratory, The Knolls, Schenectady, New York 12301

Szent-Györgyi, Albert, 1956 (14), Institute for Muscle Research, P. O. Box 187, Woods Hole, Massachusetts 02543

Taliaferro, William Hay, 1940 (10), Argonne National Laboratory, 9700 South Cass Avenue, Argonne, lllinois 60440

Tarbell, Dean Stanley, 1959 (5), Department of Chemistry, University of Rochester, Rochester, New York 14627

Tatum, Edward Lawrie, 1952 (14), Rockefeller Institute, New York, New York 10021

Taube, Henry, 1959 (5), Department of Chemistry, Stanford University, Stanford, California 94305

Teller, Edward, 1948 (3), Lawrence Radiation Laboratory, University of California, Berkeley, California 94720

Terman, Frederick Emmons, 1946 (4), Provost's Office, Stanford University, Stanford, California 94305

Thimann, Kenneth Vivian, 1948 (7), The Biological Laboratories, Harvard University, 16 Divinity Avenue, Cambridge, Massachusetts 02138

Thomas, Charles Allen, 1948 (4), Monsanto Chemical Company, 800 North Lindbergh Boulevard, St. Louis, Missouri 63166

Thomas, Llewellyn Hilleth, 1958 (3), Watson Scientific Laboratory, 612 West 115th Street, New York, New York 10025

Thomas, Tracy Yerkes, 1941 (1), Department of Mathematics, Indiana University, Bloomington, Indiana 47405

Tillett, William Smith, 1951 (10), New York University School of Medicine, 550 First Avenue, New York, New York 10016

Timoshenko, Stephen Prokop, 1940 (4), 536 West Crescent Drive, Palo Alto, California 94301

Tishler, Max, 1953 (5), Merck \& Co., Inc., Rahway, New Jersey 07065

Tousey, Richard, 1960 (2), United States Naval Research Laboratory (Codt 7140), Washington, D. C. 20390

Townes, Charles Hard, 1956 (3), Provost's Office, Massachusetts Institute of Technology, Cambridge, Massachusetts 02139

Tukey, John Wilder, 1961 (1), Fine Hall, Princeton University, P. O. Box 708, Princeton, New Jersey 08540

Turner, Francis John, 1956 (6), Department of Geology and Geophysics, University of California, Berkeley, California 94720

Turner, Richard Baldwin, 1964 (5), Department of Chemistry, Rice University, Houston, Texas 77001

Tuve, Merle Antony, 1946 (3), Department of Terrestrial Magnetism, Carnegie Institution of Washington, 5241 Broad Branch Road, N. W., Washington, D. C. 20015

Twitty, Victor Chandler, 1950 (8), Department of Biological Sciences, Stanford University, Stanford, California 94305 
Uhlenbeck, George Eugene, 1955 (3), Rockefeller Institute, New York, New York 10021

Urey, Harold Clayton, 1935 (5), University of California, San Diego, La Jolla, California 92038

Van Allen, James Alfred, 1959 (13), Department of Physics and Astronomy, State University of Iowa, Iowa City, Iowa 52240

Van Niel, Cornelis Bernardus, 1945 (7), Hopkins Marine Station of Stanford University, Pacific Grove, California 93950

Van Slyke, Donald Dexter, 1921 (14), Brookhaven National Laboratory, Upton, Long Island, New York 11973

Van Vleck, John Hasbrouck, 1935 (3), Lyman Laboratory of Physics, Harvard University, Cambridge, Massachusetts 02138

Verhoogen, John, 1956 (13), Department of Geology and Geophysics, University of California, Berkeley, California 94720

Vestine, Ernest Harry, 1954 (13), Rand Corporation, 1700 Main Street, Santa Monica, California 90401

Vickery, Hubert Bradford, 1943 (14), Connecticut Agricultural Experiment Station, New Haven, Connecticut 06504

Villard, Oswald Garrison, Jr., 1958 (13), Radioscience Laboratory, Stanford University, Stanford, California 94305

Visscher, Maurice Bolks, 1956 (9), Department of Physiology, University of Minnesota, Minneapolis, Minnesota 55455

von Békésy, Georg, 1956 (12), Memorial Hall, Harvard University, Cambridge, Massachusetts 02138

Waksman, Selman Abraham, 1942 (10), Institute of Microbiolozy, Rutgers, The State University, New Brunswick, New Jersey 08901

Wald, George, 1950 (14), The Biological Laboratories, Harvard University, 16 Divinity Avenue, Cambridge, Massachusetts 02138

Walker, John Charles, 1945 (7), College of Agriculture, University of Wisconsin, Madison, Wisconsin 53706

Wall, Frederick Theodore, 1961 (5), Department of Chemistry, University of California, Santa Barbara, California 93018

Walling, Cheves Thomson, 1964 (5), Department of Chemistry, Columbia University, New York, New York 10027

Walsh, Joseph Leonard, 1936 (1), Widener 474, Harvard University, Cambridge, Massachusetts 02138

Warner, John Christian, 1956 (5), Carnegie Institute of Technology, Pittsburgh, Pennsylvania 15213

Warren, Shields, 1962 (10), Cancer Research Institute, New England Deaconess Hospital, 194 Pilgrim Road, Boston, Massachusetts 02215

Washburn, Sherwood Larned, 1963 (11), Department of Anthropology, University of California, Berkeley, California 94720

Waters, Aaron Clement, 1964 (6), Department of Geology, University of California, Santa Barbara, California 93018

Watson, Cecil James, 1959 (10), Department of Medicine, University of Minnesota Hospital, Minneapolis, Minnesota 55455

Watson, James Dewey, 1962 (14), The Biological Laboratories, Harvard University, 16 Divinity Avenue, Cambridge, Massachusetts 02138

Webster, David Locke, 1923 (3), 1830 Cowper Street, Palo Alto, California 94301

Weinberg, Alvin Martin, 1961 (3), Oak Ridge National Laboratory, P. O. Box X, Oak Ridge, Tennessee 37831 
Weiss, Paul Alfred, 1947 (8), Graduate School of Biomedical Sciences, University of Texas, Houston, Texas 77025

Weisskopf, Victor Frederick, 1952 (3), CERN, Geneva 23, Switzerland

Weller, Thomas Huckle, 1964 (10), Department of Tropical Public Health, Harvard School of Public Health, 25 Shattuck Street, Boston, Massachusetts 02115

Went, Frits Warmolt, 1947 (7), Department of Botany, Washington University, St. Louis, Missouri 63130

Wentzel, Gregor, 1959 (3), The Enrico Fermi Institute for Nuclear Studies, University of Chicago, Chicago, Illinois 60637

Westheimer, Frank Henry, 1954 (5), Department of Chemistry, Harvard University, 12 Oxford Street, Cambridge, Massachusetts 02138

Wetmore, Alexander, 1945 (8), Smithsonian Institution, Washington, D. C. 20560

Wetmore, Ralph Hartley, 1954 (7), The Biological Laboratories, Harvard University, 16 Divinity Avenue, Cambridge, Massachusetts 02138

Wever, Ernest Glen, 1940 (12), 1514 Green Hall, Princeton University, Princeton, New Jersey 08540

Wheeler, John Archibald, 1952 (3), Palmer Physical Laboratory, Princeton University, Princeton, New Jersey 08540

Whipple, Fred Lawrence, 1959 (2), Astrophysical Observatory, Smithsonian Institution, 60 Garden Street, Cambridge, Massachusetts 02138

Whipple, George Hoyt, 1929 (10), School of Medicine and Dentistry, University of Rochester, 260 Crittenden Boulevard, Rochester, New York 14620

Whitford, Albert Edward, 1954 (2), Lick Observatory, Mount Hamilton, California 95140

Whitney, Hassler, 1945 (1), The Institute for Advanced Study, Princeton, New Jersey 08540

Whyburn, Gordon Thomas, 1951 (1), Department of Mathematics, University of Virginia, Charlottesville, Virginia 22903

Wick, Gian-Carlo, 1963 (3), Department of Physics, Brookhaven National Laboratory, Upton, Long Island, New York 11973

Wiesner, Jerome Bert, 1960 (4), Massachusetts Institute of Technology, Cambridge, Massachusetts 02139

Wigner, Eugene Paul, 1945 (3), Fine Hall, Princeton University, Princeton, New Jersey 08540

Wilder, Raymond Louis, 1963 (1), Department of Mathematics, University of Michigan, Ann Arbor, Michigan 48104

Willey, Gordon Randolph, 1960 (11), Peabody Museum, Harvard University, Cambridge, Massachusetts 02138

Williams, Carroll Milton, 1960 (8), The Biological Laboratories, Harvard University, 16 Divinity Avenue, Cambridge, Massachusetts 02138

Williams, Howel, 1950 (6), Department of Geology and Geophysics, University of California, Berkeley, California 94720

Williams, John Harry, 1961 (3), School of Physics, University of Minnesota, Minneapolis, Minnesota 55455

Williams, John Warren, 1952 (5), Department of Chemistry, University of Wisconsin, Madison, Wisconsin 53706

Williams, Robert R., 1945 (5), 45 Woodland Avenue, Summit, New Jersey 07901

Williams, Robley Cook, 1955 (14), Virus Laboratory, University of California, Berkeley, California 94720 
Williams, Roger John, 1946 (14), Clayton Foundation Biochemical Institute, University of Texas, Austin, Texas 78712

Willier, Benjamin Harrison, 1945 (8), Department of Biology, Johns Hopkins University, Baltimore, Maryland 21218

Wilson, David Wright, 1955 (14), Department of Biochemistry, School of Medicine, University of Pennsylvania, Philadelphia, Pennsylvania 19104

Wilson, Edgar Bright, Jr., 1947 (5), Department of Chemistry, Harvard University, 12 Oxford Street, Cambridge, Massachusetts 02138

Wilson, Edwin Bidwell, 1919 (3), 42 Brington Road, Brookline, Massachusetts 02146

Wilson, Olin Chaddock, 1960 (2), Mount Wilson and Palomar Observatories, 813 Santa Barbara Street, Pasadena, California 91106

Wilson, Perry William, 1955 (7), Department of Bacteriology, University of Wisconsin, Madison, Wisconsin 53706

Wilson, Robert Erastus, 1947 (4), The Westchester, 4000 Cathedral Avenue, N. W., Washington, D. C. 20016

Wilson, Robert Rathbun, 1957 (3), Laboratory of Nuclear Studies, Cornell University, Ithaca, New York 14850

Winstein, Saul, 1955 (5), Department of Chemistry, University of California, Los Angeles, California 90024

Wintersteiner, Oskar, 1950 (14), Squibb Institute for Medical Research, New Brunswick, New Jersey 08903

Wolfrom, Melville Lawrence, 1950 (14), Department of Chemistry, The Ohio State University, 88 West 18th Avenue, Columbus, Ohio 43210

Wolman, Abel, 1963 (4), 513 Ames Hall, Johns Hopkins University, Baltimore, Maryland 21218

Wood, Harland Goff, 1953 (14), Department of Biochemistry, School of Medicine, Western Reserve University, Cleveland, Ohio 44106

Wood, William Barry, Jr., 1959 (10), School of Medicine, Johns Hopkins University, 725 North Wolfe Street, Baltimore, Maryland 21205

Woodring, Wendell Phillips, 1946 (6), United States National Museum, Washington, D. C. 20560

Woodward, Robert Burns, 1953 (5), Department of Chemistry, Harvard University, 12 Oxford Street, Cambridge, Massachusetts 02138

Woolley, Dilworth Wayne, 1952 (14), Rockefeller Institute, New York, New York 10021

Woolsey, Clinton Nathan, 1960 (9), Laboratory of Neurophysiology, 283 Medical Sciences Building, University of Wisconsin, Madison, Wisconsin 53706

Wright, Sewall Green, 1934 (8), Department of Genetics, University of Wisconsin, Madison, Wisconsin 53706

Wu, Chien-Shiung, 1958 (3), Department of Physics, Columbia University, New York, New York 10027

Wulf, Oliver Reynolds, 1949 (13), United States Weather Bureau, Gates and Crellin Laboratories of Chemistry, California Institute of Technology, Pasadena, California 91109

Wyckoff, Ralph Walter Graystone, 1949 (5), Department of Physics, University of Arizona, Tucson, Arizona 85721

Yoder, Hatten Schuyler, Jr., 1958 (6), Geophysical Laboratory, Carnegie Institution of Washington, 2801 Upton Street, N. W., Washington, D. C. 20008

Yost, Don Merlin Lee, 1944 (5), California Institute of Technology, Pasadena, California 91109 
Young, William Gould, 1951 (5), Department of Chemistry, University of California, Los Angeles, California 90024

Zacharias, Jerrold Reinach, 1957 (3), Department of Physics, Massachusetts Institute of Technology, Cambridge, Massachusetts 02139

Zachariasen, Frederik William Houlder, 1949 (3), Division of the Physical Sciences, University of Chicago, Chicago, Illinois 60637

Zariski, Oscar, 1944 (1), Department of Mathematics, Harvard University, 2 Divinity Avenue, Cambridge, Massachusetts 02138

Zener, Clarence Melvin, 1959 (4), Westinghouse Research Laboratories, Beulah Road, Churchill Borough, Pittsburgh, Pennsylvania 15235

Zimm, Bruno Hasbrouck, 1958 (5), Department of Chemistry, University of California, San Diego, La Jolla, California 92038

Zinn, Walter Henry, 1956 (4), Combustion Engineering, Inc., P. O. Box 500, Windsor, Connecticut 06095

Zirkle, Raymond Elliott, 1959 (8), Department of Biophysics, University of Chicago, 5640 Ellis Avenue, Chicago, Illinois 60637

Zworykin, Vladimir Kosma, 1943 (4), RCA Laboratories, David Sarnoff Research Center, Princeton, New Jersey 08541

Zygmund, Antoni, 1960 (1), Department of Mathematics, University of Chicago, Chicago, Illinois 60637

Number of Members July 1, 1964: 697

\section{MEMBERS EMERITI}

Albright, Fuller, 1952 (10), 271 Goddard Avenue, Brookline, Massachusetts 02146

Bailey, Irving Widmer, 1929 (7), Harvard University Herbarium, 22 Divinity Avenue, Cambridge, Massachusetts 02138

Coble, Arthur Byron, 1924 (1), Lykens Hotel, Lykens, Pennsylvania 17048

Cole, Rufus, 1922 (10), Mt. Kisco, New York 10549

Coolidge, William David, 1925 (3), 1480 Lenox Road, Schenectady, New York 12308

Hartman, Carl Gottfried, 1937 (8), 219 Norwood Avenue, North Plainfield, New Jersey 07060

Kelley, Walter Pearson, 1943 (6), 108 Hilgard Hall, University of California, Berkeley, California 94720

Schultz, Adolph Hans, 1939 (11), Anthropologisches Institut, Künstlergasse 15, Zurich, Switzerland

Tyzzer, Ernest Edward, 1942 (10), 484 Water Street, Wakefield, Massachusetts 01880

Vandiver, Harry Shultz, 1934 (1), Box 7881, University of Texas, Austin, Texas 78712

Number of Members Emeriti July 1, 1964: 10 


\section{FOREIGN ASSOCIATES}

The number in parentheses following the year of election indicates association within the Sections of the National Academy of Sciences.

Adrian, of Cambridge, Edgar Douglas, Baron, 1941 (9), Trinity College, Cambridge, England

Alexandroff, Paul A., 1947 (1), Mathematical Institute of the Academy of Sciences of the U. S. S. R., Bolshaya Kalushskaya 19, Moscow, U. S. S. R.

Amaldi, Edoardo, 1962 (3), University of Rome, Piazzale delle Scienze, 5, Rome, Italy

Ambartsumian, Victor Amazaspovich, 1959 (2), Burakan Astronomical Observatory, Erevan, Armenia, U. S. S. R.

Andrewes, Sir Christopher Howard, 1964 (10), Overchalke, Coombe Bissett, Salisbury, Wiltshire, England

Bailey, Sir Edward, 1944 (6), 76 Hampstead Way, London, N. W. 11, England

Bartlett, Sir Frederic Charles, 1947 (12), 161 Huntingdon Road, Cambridge, England

Best, Charles Herbert, 1950 (9), Banting and Best Department of Medical Research, University of Toronto, Toronto, Canada

Bhabha, Homi Jehangir, 1963 (3), Tata Institute of Fundamental Research, Apollo Pier Road, Bombay 1, India

Born, Max, 1955 (3), Marcard Strasse 4, Bad Pyrmont, Germany

Bragg, Sir William Lawrence, 1945 (3), The Royal Institution, 21 Albemarle Street, London, W. 1., England

de Broglie, Prince Louis, 1948 (3), 94 Rue Perronet, Neuilly-sur-Seine, France

Brun, Edmond Antoine, 1960 (4), University of Paris, 8-10, place du Commerce, Paris XV, France

Bullard, Sir Edward Crisp, 1959 (13), Madingley Rise, Madingley Road, Cambridge, England

Bullen, Keith Edward, 1961 (13), Department of Applied Mathematics, University of Sydney, Sydney, Australia

Burnet, Sir Macfarlane, 1954 (10), The Walter and Eliza Hall Institute of Medical Research, Melbourne, Australia

Caso, Alfonso, 1943 (11), Avenida Central 234, Tlacopac, Villa Obregón, Mexico 20, D. F.

Chapman, Sydney, 1946 (13), High Altitude Observatory, Boulder, Colorado 80301, U.S. A.

Clark, Sir Wilfrid Le Gros, 1963 (11), Department of Human Anatomy, Oxford University, South Parks Road, Oxford, England

Dale, Sir Henry Hallett, 1940 (9), The Wellcome Trust, 52 Queen Anne Street, London, W. 1, England

Dirac, Paul Adrien Maurice, 1949 (1), Department of Mathematics, St. John's College, Cambridge, England

Ephrussi, Boris, 1961 (7), Developmental Biology Center, Western Reserve University, 2127 Cornell Road, Cleveland, Ohio 44106, U. S. A.

Eskola, Pentti Eelis, 1951 (6), Helsinki University, Snellmanink. 5, Helsinki, Finland

Florey, Sir Howard Walter, 1963 (10), The Royal Society, Burlington House, London, W. 1, England 
von Frisch, Karl, 1951 (8), The Zoological Institute, University of Munich, Munich, Germany

Geijer, Per, 1958 (6), Agnevaegen 5, Djursholm I, Sweden

Haldane, John Burdon Sanderson, 1964 (8), Genetics and Biometry Laboratory of the Government of Orissa, Bhubaneswar-3, Orissa, India

Heisenberg, Werner, 1961 (3), Max Planck Institut für Physik und Astrophysik, Aumeisterstrasse 6, Munich 23, Germany

Hill, Archibald Vivian, 1941 (9), 16 Bishopswood Road, Highgate, London, N. 6, England

Hinshelwood, Sir Cyril Norman, 1960 (5), Department of Chemistry (Room 41), Imperial College, Imperial Institute Road, London, S.W. 7, England

Hodge, Sir William Vallance Douglas, 1959 (1), The Master's Lodge, Pembroke College, Cambridge, England

Hopf, Heinz, 1957 (1), Swiss Federal Institute of Technology, Zurich, Switzerland

Houssay, Bernardo Alberto, 1940 (9), Viamonte 2790, Buenos Aires, Argentina

Jeffreys, Sir Harold, 1945 (13), St. John's College, Cambridge, England

Kapitza, Peter Leonidovich, 1946 (3), S. I. Vavilov Institute of Physical Problems, Academy of Sciences of the U. S. S. R., Moscow, U. S. S. R.

Karrer, Paul, 1945 (5), Spyristeig 30, Zurich 7, Switzerland

Kihara, Hitoshi, 1958 (7), National Institute of Genetics, Misima, Japan

Konorski, Jerzy, 1963 (12), Department of Neurophysiology, Nencki Institute of Experimental Biology, 3, Pasteur Street, Warsaw 22, Poland

Krebs, Sir Hans Adolf, 1964 (14), Department of Biochemistry, Oxford University, South Parks Road, Oxford, England

Kuno, Hisashi, 1963 (6), Geological Institute, University of Tokyo, Tokyo, Japan

Landau, Lev Davidovich, 1960 (3), S. I. Vavilov Institute of Physical Problems, Academy of Sciences of the U. S. S. R., Moscow, U. S. S. R.

Leloir, Luis F., 1960 (14), Instituto de Investigaciones Bioquimicas, Obligado 2490, Buenos Aires, Argentina

Levi, Giuseppe, 1940 (8), Instituto di Anatomia Umana, Corso Massimo D’Azeglio, 52 , Turin, Italy

Lim, Robert K. S., 1942 (9), Medical Sciences Research Laboratory, Miles Laboratories, Inc., Elkhart, Indiana 46514, U. S. A.

Lindblad, Bertil, 1955 (2), Stockholm Observatory, Saltsjöbaden, Sweden

Lundegårdh, Henrik Gunnar, 1964 (7), Research Laboratory in Plant Physiology, Penningby, Sweden

Lwoff, André, 1955 (9), Institut Pasteur, Paris XV, France

Lynen, Feodor, 1962 (14), Max-Planck-Institut für Zellchemie, Karlstrasse 23-25, Munich 2, Germany

Michotte, Albert Edouard (Baron Michotte van den Berck), 1956 (12), University of Louvain, Louvain, Belgium

Minnaert, Marcel Gilles Jozef, 1964 (2), Utrecht Observatory, Zonnenburg 2, Utrecht, The Netherlands

Mott, Sir Nevill Francis, 1957 (3), University of Cambridge, Cavendish Laboratory, Free School Lane, Cambridge, England

Oort, Jan Hendrik, 1953 (2), Observatory of Leiden, Leiden, The Netherlands

Penfield, Wilder, 1953 (10), Montreal Neurological Institute, 3801 University Street, Montreal 2, Canada

Penney, Sir William George, 1962 (4), United Kingdom Atomic Energy Authority, Charles II Street, London, S. W. 1, England

Piéron, Henri, 1949 (12), Institut de Psychologie, 28, rue Serpente, Paris VI, France 
Prelog, Vladimir, 1961 (5), Laboratorium für organische Chemie, Eidgenossische Technische Hochschule, Zurich, Switzerland

Reichstein, Tadeus, 1952 (5), Organisch-chemische Anstalt, St. Johanns-Ring 19, Basel, Switzerland

Robertson, Rutherford Ness, 1962 (7), Department of Botany, University of Adelaide, Adelaide, South Australia

Robinson, Sir Robert, 1934 (5), 170 Piccadilly, London, W. 1, England

Roy, Maurice, 1964 (4), 86, Avenue Niel, Paris XVII, France

Ruzicka, Leopold, 1944 (5), Department of Organic Chemistry, Institute of Technology, Zurich, Switzerland

Semenov, Nikolai Nikolaevich, 1963 (5), Institute of Chemical Physics, Vorobyevskoye chaussee 2, Moscow, V-133, U. S. S. R.

Southwell, Sir Richard Vynne, 1943 (4), The Old House, Trumpington, Cambridge, England

Svedberg, The, 1945 (5), Fysikalisk-Kemiska Institutionen, Uppsala University, Uppsala, Sweden

Taylor, Sir Geoffrey Ingram, 1945 (1), Trinity College, Cambridge, England

Theorell, Axel Hugo, 1957 (14), Nobel Institute of Medicine, Solnavagen 1, Stockholm 60, Sweden

Tiselius, Arne W. K., 1949 (14), Institute of Biochemistry, Uppsala University, Uppsala, Sweden

Todd, of Trumpington, Alexander Robertus, Baron, 1955 (5), University of Cambridge, University Chemical Laboratory, Lensfield Road, Cambridge, England

Vening Meinesz, Felix Andries, 1939 (13), Potgieterlaan 5, Amersfoot, The Netherlands

Watson, D. M. S., 1938 (8), University College, Gower Street, London, W. C. 1, England

Winge, Öjvind, 1949 (14), Department of Physiology, Carlsberg Laboratory, Copenhagen (Valby), Denmark

Yukawa, Hideki, 1949 (3), Yukawa Hall, Kyoto Unniversity, Kyoto, Japan

Number of Foreign Associates July 1, 1964: 72 


\section{SECTIONS}

\section{(1) Mathematics -47 members}

Brauer, * Richard, Chairman (1967)

Ahlfors, L. V.

Albert, A. A.

Alexander, J. W.

Bers, Lipman

Bochner, S.

Bott, Raoul

Chern, S. S.

Courant, R.

Doob, J. L.

Douglas, Jesse

Eilenberg, S.

Eisenhart, L. P.

Evans, G. C.

Feller, William
Friedrichs, K. O.

Gödel, Kurt

Hille, Einar

Jacobson, Nathan

John, Fritz

Lefschetz, Solomon

Lewy, Hans

Lin, C. C.

Mackey, George W.

Mac Lane, Saunders

McShane, E. J.

Milnor, J. W.

Montgomery, Deane

Moore, R. L.

Morrey, C. B., Jr.

Morse, Marston

\section{Foreign Associates}

Alexandroff, P. A.

Dirac, P. A. M.
Hodge, Sir William Hopf, Heinz
Murnaghan, F. D.

Neyman, Jerzy

Shannon, C. E.

Smith, Paul A.

Spencer, D. C.

Steenrod, N. E.

Stoker, J. J.

Stone, M. H.

Thomas, T. Y.

Tukey, John W.

Walsh, J. L.

Whitney, Hassler

Whyburn, G. T.

Wilder, R. L.

Zariski, O.

Zygmund, Antoni

* In Dr. Brauer's absence from the country during the coming year, Hassler

Whitney will serve as acting chairman.

\section{(2) Astronomy-30 members}

Menzel, D. H., Chairman (1965)

Abbot, C. G.

Aller, L. H.

Babcock, H. D.

Babcock, H. W.

Bowen, I. S.

Brouwer, Dirk

Chandrasekhar, S.

Clemence, G. M.

Goldberg, Leo

Ambartsumian, V.

Lindblad, Bertil
Greenstein, J. L.

Herbig, George H.

Herget, Paul

Joy, A. H.

Kuiper, G. P.

Mayall, N. U.

Minkowski, R. L.

Morgan, W. W.

Sandage, A. R.

Schwarzschild, M.

\section{Foreign Associates}

Minnaert, M. G. J.

(3) Physics -97 members

Anderson, C. D., Chairman (1966)

Allison, S. K.

Alvarez, L. W.

Anderson, H. L.
Bacher, R. F.

Bainbridge, K. T.

Bardeen, John

Beams, J. W.

Bethe, H. A.
Seares, F. H.

Shane, C. D.

Shapley, Harlow

Slipher, V. M.

Spitzer, Lyman, Jr.

Stebbins, Joel

Tousey, Richard

Whipple, F. L.

Whitford, A. E.

Wilson, Olin C.

Oort, Jan Hendrik

Birge, R. T.

Bleakney, Walker

Bloch, Felix

Bloembergen, N.

Bradbury, N. E. 
Brattain, W. H.

Breit, Gregory

Brillouin, Leon

Brode, R. B.

-Chamberlain, Owen

Chew, Geoffrey F.

Condon, E. U.

Dennison, D. M.

Deutsch, M.

DuBridge, L. A.

DuMond, J. W. M.

Dunning, J. R.

Dyson, Freeman J.

Epstein, P. S.

Fairbank, W. M.

Feynman, R. P.

Fowler, W. A.

Gamow, George

Gell-Mann, Murray

- Glaser, Donald A.

Goldberger, M. L.

Goldhaber, M.

Gordy, Walter

Goudsmit, S. A.

Herb, R. G.

Herzfeld, K. F.

Hofstadter, R.

Houston, W. V.

Amaldi, Edoardo

Bhabha, Homi J.

Born, Max

Bragg, Sir Lawrence
Hull, A. W.

Inghram, Mark G.

Kemble, E. C.

Kerst, D. W.

Kittel, C.

Kusch, P.

Lamb, W. E., Jr.

Land, E. H.

Lauritsen, C. C.

Lee, Tsung-Dao

Loomis, F. W.

Marshak, R. E.

- Mayer, M. G.

McMillan, E. M.

Meggers, W. F.

Morse, P. M.

Mulliken, R. S.

Nier, A. O. C.

O'Brien, Brian

Oppenheimer, J. R.

Pais, A.

Panofsky, W. K. H.

Pound, R. V.

Purcell, E. M.

Rabi, I. I.

Ramsey, N. F.

Rossi, Bruno

Schiff, L. I.

\section{Foreign Associates}

de Broglie, Prince Louis

Heisenberg, Werner

Kapitza, P. L.
Schwinger, Julian

- Segrè, E.

Seitz, Frederick

Serber, R.

Simpson, J. A.

Slater, J. C.

Stern, Otto

Street, J. C.

- Teller, Edward

Thomas, L. H.

Townes, C. H.

Tuve, M. A.

Uhlenbeck, G. E.

Van Vleck, J. H.

Webster, D. L.

Weinberg, Alvin M.

Weisskopf, V. F.

Wentzel, Gregor

Wheeler, J. A.

Wick, Gian-Carlo

Wigner, E. P.

Williams, John $\mathrm{H}$.

Wilson, Edwin B.

Wilson, Robert R.

Wu, C. S.

Zacharias, J. R.

Zachariasen, W. H.

\section{(4) Engineering -52 members}

Sherwood, T. K., Chair- , Edgerton, H. E. man (1965)

Astin, A. V.

Bain, E. C.

Benedict, Manson

Bode, H. W.

Brooks, Harvey

Bush, Vannevar

Chipman, John

Curme, G. O., Jr.

Darken, L. S.

Den Hartog, J. P.

Draper, C. S.

Dryden, H. L.

\section{Fisk, J. B.}

Fletcher, Harvey

Foote, P. D.

Gibbs, W. F.

Gilliland, E. R.

Greenewalt, C. H.

Hoover, Herbert

Hottel, H. C.

Hunsaker, J. C.

Jeffries, Zay

Kelly, M. J.

Kinzel, A. B.

Lewis, W. K.
Loomis, A. L.

Mehl, R. F.

Millikan, Clark B.

Olson, Harry F.

Pickering, W. H.

Pierce, J. R.

Piore, E. R.

Raymond, A. E.

Savage, J. L.

Shockley, W.

Slepian, Joseph

Smith, C. S.

Soderberg, C. R.

Sporn, Philip 
Stratton, J. A.

Suits, C. G.

Terman, F. E.

Thomas, C. A.

Brun, Edmond A.

Penney, Sir William

\section{(5) Chemistry - 100 members}

Crawford, Bryce, Jr., Chairman (1968)

Adams, Roger

Badger, R. M.

Baker, W. O.

Bartlett, P. D.

Blomquist, A. T.

Boekelheide, V.

Bolton, E. K.

Brewer, Leo

Brode, W. R.

Brown, H. C.

Calvin, Melvin

Conant, J. B.

Connick, R. E.

Cope, A. C.

Cram, Donald J.

Curtin, David Y.

Daniels, Farrington

Davidson, N.

Debye, Peter

Djerassi, Carl

Doering, William

Elderfield, R. C.

Emmett, P. H.

Eyring, Henry

Ferry, John D.

Fieser, L. F.

Flory, P. J.

Folkers, Karl

Fuoss, R. M.

Fuson, R. C.

Gates, M.

Giauque, W. F.

Hinshelwood, Sir Cyril

Karrer, Paul

Prelog, Vladimir
Timoshenko, Stephen

Wiesner, J. B.

Wilson, Robert E.

Wolman, Abel

Foreign Associates

Roy, Maurice

Southwell, Sir Richard

Zener, Clarence

Zinn, W. H.

Zworykin, V. K.
Gilman, Henry

Gutowsky, H. S.

Hammett, L. P.

Hammond, G. S.

Harned, H. S.

Hauser, C. R.

Hildebrand, J. H.

Hirschfelder, J. O.

Hornig, D. F.

Hutchison, C. A., Jr.

Jacobs, W. A.

Johnson, J. R.

Johnson, W. S.

Kauzmann, Walter

Keyes, F. G.

Kimball, G. E.

Kistiakowsky, G. B.

Kolthoff, I. M.

Kraus, C. A.

La Mer, V. K.

Leonard, N. J.

Libby, W. F.

Lind, S. C.

Lipscomb, W. N.

Long, Franklin A.

Longsworth, L. G.

MacInnes, D. A.

Mark, H. F.

Marvel, C. S.

Mayer, J. E.

McElvain, S. M.

Newman, M. S.

Noyes, W. A., Jr.

Onsager, Lars

\section{Foreign Associates}

Reichstein, Tadeus

Robinson, Sir Robert

Ruzicka, Leopold
Pauling, Linus

Perlman, Isadore

Pitzer, K. S.

Rice, Oscar K.

Roberts, John D.

Rossini, F. D.

Scatchard, George

Seaborg, G. T.

Shedlovsky, Theodore

Sheehan, J. C.

Smith, L. I.

Smyth, C. P.

Spedding, F. H.

Stockmayer, W. H.

Stork, Gilbert

Tarbell, D. S.

Taube, Henry

Tishler, Max

Turner, Richard B.

Urey, H. C.

Wall, Frederick T.

Walling, Cheves

Warner, J. C.

Westheimer, F. H.

Williams, J. W.

Williams, Robert R.

Wilson, E. Bright, Jr.

Winstein, Saul

Woodward, R. B.

Wyckoff, R. W. G.

Yost, D. M.

Young, W. G.

Zimm, B. H.

Semenov, Nikolai N.

Svedberg, The

Lord Todd 
(6) Geology -38 members

Anderson, C. A., Chairman (1966)

Abelson, P. H.

Allen, E. T.

Birch, Francis

Blackwelder, Eliot

Bradley, W. H.

Bramlette, M. N.

Bucher, W. H.

Buddington, A. F.

Buerger, M. J.

Chaney, R. W.

Cloos, Ernst

Bailey, Sir Edward

Eskola, Pentti

Thimann, K. V., Chairman (1965)

Anderson, Edgar

Anderson, T. F.

Arnold, William A.

Arnon, Daniel I.

Barker, H. A.

Beadle, G. W.

Benzer, Seymour

Blinks, L. R.

Bonner, James

Borthwick, H. A.

Braun, A. C.

Brink, R. A.

Burkholder, P. R.

Burris, R. H.

Chandler, W. H.

Ephrussi, Boris

Kihara, H.
Cloud, Preston, E., Jr.

Dunbar, C. O.

Garrels, R. M.

Gilluly, James

Gregory, W. K.

Hedberg, H. D.

Hess, H. H.

Hewett, D. F.

Hubbert, M. King

James, Harold L.

Knopf, Adolph

Krauskopf, K. B.

Longwell, C. R.

\section{Foreign Associates}

Geijer, Per

\section{(7) Botany -50 members}

Clausen, Jens

Cleland, R. E.

Couch, J. N.

Delbrück, Max

Esau, Katherine

Fred, E. B.

French, C. S.

Goddard, D. R.

Hendricks, S. B.

Hershey, A. D.

Hollaender, A.

Horsfall, J. G.

Kaufmann, B. P.

Kramer, Paul J.

Mangelsdorf, P. C.

McClintock, Barbara

Raper, John R.

\section{Foreign Associates}

Lundegårdh, H. G.
Lovering, T. S.

Mackin, J. H.

Nolan, T. B.

Patterson, Bryan

Rubey, W. W.

Russell, R. J.

Schairer, J. F.

Simpson, G. G.

Turner, F. J.

Waters, Aaron C.

Williams, Howel

Woodring, W. P.

Yoder, H. S., Jr.

Kuno, Hisashi

Raper, K. B.

Rhoades, M. M.

Riker, A. J.

Robbins, W. J.

Roberts, R. B.

Sax, Karl

Sears, Ernest R.

Sinnott, E. W.

Skoog, Folke

Smith, A. C.

Stakman, E. C.

Stebbins, G. L.

Van Niel, C. B.

Walker, J. C.

Went, F. W.

Wetmore, R. H.

Wilson, P. W.

Robertson, R. N.

(8) Zoology and Anatomy-65 members

Glass, H. Bentley, Chairman (1967)

Bartelmez, G. W.

Bigelow, H. B.

Bloom, William
Bodenstein, D. H.

Bodian, David

Briggs, Robert

Bullock, T. H.

Burns, R. K.
Cleveland, L. R.

Colbert, E. H.

Corner, G. W.

Crow, James $F$.

Danforth, C. H. 
Darlington, P. J., Jr.

Demerec, Milislav

Dobzhansky, Th.

Dunn, L. C.

Emerson, Alfred E.

Flexner, Louis B.

Friedmann, Herbert

Griffin, D. R.

Hamburger, Viktor

Haskins, C. P.

Hisaw, F. L.

Holtfreter, J.

Hubbs, C. L.

Hutchinson, G. E.

Hyman, Libbie

Irwin, M. R.

Jacobs, M. H.

von Frisch, Karl

Haldane, J. B. S.
Kellogg, Remington

Lerner, I. M.

Lewis, W. H.

Mayr, Ernst

Mazia, Daniel

Metz, C. W.

Miller, A. H.

Mirsky, A. E.

Moore, J. A.

Muller, H. J.

Neel, J. V.

Painter, T. S.

Palade, G. E.

Pittendrigh, C. S.

Porter, Keith R.

Riddle, Oscar

Roeder, Kenneth D.

\section{Foreign Associates}

Levi, Giuseppe
Romer, A. S.

Schmidt-Nielsen, K.

Schmitt, F. O.

Scholander, P. F.

Smith, Philip E.

Sonneborn, T. M.

Stern, Curt

Stone, W. S.

Straus, W. L., Jr.

Sturtevant, A. H.

Twitty, V. C.

Weiss, Paul

Wetmore, Alexander

Williams, C. M.

Willier, B. H.

Wright, Sewall

Zirkle, R. E.

Watson, D. M. S.

\section{(9) Physiology -34 members}

Davis, Hallowell, Chairman (1966)

Astwood, E. B.

Aub, Joseph C.

Bard, Philip

Brink, Frank, Jr.

Bronk, Detlev W.

Cole, K. S.

Comroe, Julius H., Jr.

Cournand, André

Erlanger, Joseph

Evans, H. M.

Lord Adrian

Best, C. H.

Dale, Sir Henry
Fenn, W. O.

Forbes, Alexander

Gerard, R. W.

Gilman, Alfred

Hartline, H. K.

Hastings, A. B.

Ingle, D. J.

Kety, Seymour S.

Krayer, Otto

Kuffler, S. W.

Landis, E. M.

Lloyd, D. P. C.

\section{Foreign Associates}

Hill, A. V.

Houssay, B. A.
Loeb, R. F.

Long, C. N. H.

Lorente de Nó, R.

Magoun, H. W.

Marshall, E. K., Jr.

Pitts, R. F.

Richards, A. N.

Richards, D. W.

Schmidt, C. F.

Visscher, M. B.

Woolsey, C. N.

(10) Pathology and Microbiology - 46 members

Francis, Thomas, Jr., Chairman (1966)

Armstrong, Charles

Bailey, P.

Blalock, Alfred

Cannon, P. R.

Castle, W. B.
Coggeshall, L. T.

Coons, Albert $\mathrm{H}$.

Dalldorf, Gilbert

Dingle, J. H.

Dragstedt, L. R.

Dubos, R. J.

Dulbecco, Renato
Eagle, Harry

Enders, J. F.

Goebel, W. F.

Heidelberger, Michael

Horsfall, F. L., Jr.

Hotchkiss, R. D.

Huebner, R. J. 
Huggins, C. B.

Little, C. C.

Long, E. R.

Luria, S. E.

MacLeod, C. M.

Maxcy, K. F.

McCarty, Maclyn

McMaster, P. D.

Meyer, K. F.

Andrewes, Sir Christopher Burnet, Sir Macfarlane

Hallowell, A. Irving, Chairman (1966)

Albright, W. F.

Braidwood, Robert J.

Coon, C. S.

Eggan, Fred

Caso, Alfonso
(11) Anthropology-15 members

Miller, C. P.

Opie, E. L.

Paul, John R.

Puck, T. T.

Rich, A. R.

Robertson, O. H.

Rous, Peyton

Sabin, Albert B.

Shope, R. E.

\section{Foreign Associates}

Florey, Sir Howard

Haury, E. W.

Lothrop, S. K.

Movius, H. L., Jr.

Murdock, G. P.

Rouse, Irving

Penfield, Wilder
Taliaferro, W. H.

Tillett, W. S.

Waksman, S. A.

Warren, Shields

Watson, C. J.

Weller, Thomas $\mathrm{H}$.

Whipple, G. H.

Wood, W. Barry, Jr.
Shapiro, H. L.

Steward, J. H.

Stewart, T. D.

Washburn, S. L.

Willey, G. R.

\section{Foreign Associates}

\section{Clark, Sir Wilfrid LeGros}

(12) Psychology -25 members

Hilgard, E. R., Chair-

Beach, F. A.

Boring, E. G.

Carmichael, Leonard

Estes, W. K.

Galambos, R.

Graham, C. H.

Guilford, J. P.

Bartlett, Sir Frederic

Konorski, Jerzy
Harlow, H. F.

Klüver, Heinrich

Köhler, Wolfgang

Lindsley, D. B.

Miles, W. R.

Miller, George A.

Miller, N. E.

Neff, William D.

Pfaffmann, Carl

\section{Foreign Associates}

Michotte, Albert E.
Richter, C. P.

Riggs, Lorrin A.

Skinner, B. F.

Spence, K. W.

Sperry, R. W.

Stevens, S. S.

v. Békésy, Georg

Wever, E. G.

\section{(13) Geophysics - 37 members}

Revelle, Roger, Chairman (1966)

Adams, L. H.

Arnold, James R.

Benioff, Hugo

Berkner, L. V.
Bjerknes, J.

Booker, H. G.

Brown, Harrison

Byerly, Perry

Byers, H. R.

Charney, Jule G.
Eckart, Carl

Elsasser, W. M.

Ewing, Maurice

Forbush, Scott E.

Friedman, H.

Griggs, D. T. 
Gunn, Ross

Haurwitz, B.

Iselin, C. O'D.

Kaplan, Joseph

Knopoff, Leon

Lambert, W. D.

MacDonald, G. J. F.

Bullard, Sir Edward

Bullen, Keith Edward
Munk, W. H.

Pekeris, C. L.

Piggot, C. S.

Press, Frank

Redfield, A. C.

Reichelderfer, F. W.

Slichter, L. B.

\section{Foreign Associates}

Chapman, Sydney

Jeffreys, Sir Harold
Stommel, Henry

Van Allen, J. A.

Verhoogen, John

Vestine, E. H.

Villard, O. G., Jr.

Wulf, O. R.

(14) Biochemistry - 61 members

Carter, H. E., Chairman (1966)

Anfinsen, C. B.

Ball, E. G.

Balls, A. K.

Bloch, K. E.

Buchanan, John M.

Chance, Britton

Clarke, H. T.

Cori, Carl F.

Craig, L. C.

Doisy, E. A.

Doty, Paul

Doudoroff, Michael

du Vigneaud, V.

Edsall, J. T.

Fruton, J. S.

Green, David E.

Handler, Philip

Hassid, W. Z.

Hofmann, Klaus

Krebs, Sir Hans A.

Leloir, Luis F.
Horecker, B. L.

Kalckar, H. M.

Kamen, Martin D.

Kendall, E. C.

Kennedy, E. P.

King, C. G.

Kornberg, A.

Lardy, Henry

Lederberg, Joshua

Lehninger, A. L.

Link, K. P.

Lipmann, Fritz

Lowry, Oliver H.

Maynard, L. A.

McCollum, E. V.

McElroy, W. D.

Moore, Stanford

Neurath, Hans

Northrop, J. H.

Ochoa, Severo

Oncley, J. L.

Foreign Associates

Lynen, Feodor

Theorell, Hugo
Rittenberg, David

Rose, W. C.

Shemin, David

Smith, Emil L.

Snell, E. E.

Stanley, W. M.

Stein, W. H.

Szent-Györgyi, A.

Tatum, E. L.

Van Slyke, D. D.

Vickery, H. B.

Wald, George

Watson, J. D.

Williams, R. C.

Williams, R. J.

Wilson, D. W.

Wintersteiner, $\mathrm{O}$.

Wolfrom, M. L.

Wood, H. G.

Woolley, D. W.

Tiselius, Arne W. K.

Winge, Öjvind 